\title{
Pion-pion scattering amplitude. III. Improving the analysis with forward dispersion relations and Roy equations
}

\author{
R. Kamiński \\ Department of Theoretical Physics, Henryk Niewodniczański Institute of Nuclear Physics, Polish Academy of Sciences, 31-342, \\ Kraków, Poland \\ J. R. Peláez \\ Departamento de Física Teórica, II (Métodos Matemáticos), Facultad de Ciencias Físicas, Universidad Complutense de Madrid, \\ E-28040, Madrid, Spain \\ F. J. Ynduráin \\ Departamento de Física Teórica, C-XI Universidad Autónoma de Madrid, Canto Blanco, E-28049, Madrid, Spain
}

(Received 2 November 2007; published 19 March 2008)

\begin{abstract}
We complete and improve the fits to experimental $\pi \pi$ scattering amplitudes, both at low and high energies, that we performed in the previous papers of this series. We then verify that the corresponding amplitudes satisfy analyticity requirements, in the form of partial wave analyticity at low energies, forward dispersion relations (FDR) at all energies, and Roy equations below $\bar{K} K$ threshold; the first by construction, the last two, inside experimental errors. Then we repeat the fits including as constraints FDR and Roy equations. The ensuing central values of the various scattering amplitudes verify very accurately FDR and, especially, Roy equations, and change very little from what we found by just fitting data, with the exception of the D2 wave phase shift, for which one parameter moves by $1.5 \sigma$. These improved parametrizations therefore provide a reliable representation of pion-pion amplitudes with which one can test various physical relations. We also present a list of low energy parameters and other observables. In particular, we find $a_{0}^{(0)}=0.223 \pm 0.009 M_{\pi}^{-1}, a_{0}^{(2)}=-0.0444 \pm 0.0045 M_{\pi}^{-1}$, and $\delta_{0}^{(0)}\left(m_{K}^{2}\right)-\delta_{0}^{(2)}\left(m_{K}^{2}\right)=$ $50.9 \pm 1.2^{\circ}$.

DOI: 10.1103/PhysRevD.77.054015

PACS numbers: 13.75.Lb, 11.55.- m, 11.55.Fv, 11.80.Et
\end{abstract}

\section{INTRODUCTION}

In two recent papers, $[1,2]$, we have presented a set of fits to the data on $\pi \pi$ scattering phase shifts and inelasticities, and we also checked how well forward dispersion relations (FDR, henceforth) are satisfied by different $\pi \pi$ scattering phase shift analyses (including our own), finding in fact that some among the more widely used sets of phase shifts failed to pass this test by several standard deviations. We then performed (in Ref. [1]) a consistent energydependent phase shift analysis of $\pi \pi$ scattering amplitudes in which we constrained the fits by requiring verification of FDR.

In the present paper we complete and improve on the results in Refs. [1,2] in various ways. First of all (Sec. II) we incorporate the fit to data in Ref. [3] for the S0 wave which is very precise thanks to use of recent experimental results [4] and a more appropriate parametrization, which provides an accurate determination of $\delta_{0}^{(0)}(s)$ below the $\bar{K} K$ threshold. The precision of this determination is such that we have to refine also the analysis of some other waves. Thus, we improve the S2 wave as given in Ref. [1] by requiring a smoother junction between the low energy $\left(s^{1 / 2} \leq 932 \mathrm{MeV}\right)$ and intermediate energy $(932 \mathrm{MeV} \leq$ $s^{1 / 2} \leq 1420 \mathrm{MeV}$ ) regions. We also (slightly) improve the fit to the inelasticity of the D0 wave, smoothing the onset of inelasticity above the $\bar{K} K$ threshold, and the parametriza- tion of the $\mathrm{P}$ and $\mathrm{F}$ waves removing their ghosts. Finally, we improve the error analysis of the D2 wave.

We also make (Sec. III) some improvements in the high energy $\left(s^{1 / 2}>1.42 \mathrm{GeV}\right)$ input, especially in the momentum transfer dependence of the amplitudes, necessary to evaluate Roy equations [5]. Indeed, for Roy equations, we need Regge formulas away from the forward direction, so we extend our analysis of the Regge amplitudes there. For the imaginary part of a scattering amplitude, $\operatorname{Im} F(s, t)$, one only expects Regge theory to be valid for $s \gg \Lambda^{2},|t| \ll s$ (with $\Lambda \simeq 0.35 \mathrm{GeV}$ the QCD parameter); this will limit the validity of the Roy equations to energies $\leqslant 1 \mathrm{GeV}$ (as a matter of fact, we here only evaluate them below $\bar{K} K$ threshold). The corresponding Regge parameters were obtained, in the forward direction, by relating the $\pi \pi$ cross section to the $\pi N$ and $N N$ cross sections, using factorization, and fitting all three processes. Away from the forward direction, we fix other parameters using also sum rules, in Secs. V and VI.

All of this (Regge parameters and partial wave amplitudes) constitute a set of pion-pion amplitudes, obtained with unconstrained fits to data, that we denote by "UFD set", which we have collected in Appendix A.

Next, in Sec. IV, we test FDR and Roy equations for this UFD set. We get good verification in both cases, to a level of average agreement corresponding to 1.00 standard deviations, for FDR, and to 0.97 standard deviations for the 
Roy equations. The fact that FDR and Roy equations are practically satisfied within errors makes it reasonable to improve the fits to data including as a constraint the verification of forward dispersion relations and Roy equations: this should provide a set of parametrizations to partial waves fully compatible with analyticity and, hence, with more reliable central values. This we do in Sec. V; here we also constrain the fits by requiring verification of two sum rules which relate higher $\left(s^{1 / 2}>1420 \mathrm{MeV}\right)$ and lower energies, and which permit us to refine the values for Regge parameters away from the forward direction. This procedure provides a set of $\pi \pi$ amplitudes, that we call constrained fit to data, or CFD set, that not only fit data, but also satisfy sum rules, FDR, and Roy equations, well within the rather small errors we now have; in particular, the degree of verification of the FDR below $932 \mathrm{MeV}$ and, especially, of the Roy equations, is spectacular. It turns out that all parameters for all waves remain inside the error bars we obtained by just fitting the data (UFD set), except for the D2 wave where, in particular, one parameter changes by $1.5 \sigma$. The result of these constrained fits is collected in Appendix B for the partial wave amplitudes and Regge parameters. As it happened in Ref. [1], we still find a marked hump in the S0 wave both for the unconstrained and constrained fits, between 400 and $900 \mathrm{MeV}$ of energy, which structure is thus shown to be compatible with both FDR and Roy equations. In Sec. VI we present the values of the low energy parameters and other observables that follow from both fits to data (UFD set) and, especially, constrained fits (CFD set). The values of some parameters are improved using sum rule determinations. In particular, for the scattering lengths for the S0, S2 waves we find the very accurate determination

$$
\begin{aligned}
& a_{0}^{(0)}=0.223 \pm 0.009 M_{\pi}^{-1}, \\
& a_{0}^{(2)}=-0.0444 \pm 0.0045 M_{\pi}^{-1} ;
\end{aligned}
$$

here, and in what follows, $M_{\pi}$ represents the mass of the charged pion, that we take $M_{\pi}=139.57 \mathrm{MeV}$. The article is finished in Sec. VII where we give the conclusions, and a short discussion of our results; particularly, comparing them with other independent evaluations (theoretical and experimental) of the $\pi \pi$ scattering amplitudes. A few words on isospin breaking corrections (which affect very little our results) for the S0, P waves are also said there.

\section{FITS TO DATA}

In the present section we briefly summarize the methods and results in Refs. [1,2], together with a few improvements, obtained fitting experimental data for $\pi \pi$ partial waves, up to the energy of $1.42 \mathrm{GeV}$. Above this energy we assume the scattering amplitudes to be given by Regge formulas, that we discuss in Sec. III below. The reason why we choose to use Regge theory above $1.42 \mathrm{GeV}$ is that, whereas there are uncertainties in the Regge expressions at the lower part of the energy range where we use them, say between $1.42 \mathrm{GeV}$ and $\sim 1.8 \mathrm{GeV}$, these are substantially smaller than the uncertainties in $\pi \pi$ phase shift analyses in the same energy region. Broadly speaking we distinguish two energy regions in the fits to experimental partial wave data: the low energy region, $s^{1 / 2} \leq s_{i}^{1 / 2}, s_{i}^{1 / 2} \sim 1 \mathrm{GeV}$, and the intermediate energy region, $s_{i}^{1 / 2} \leqslant s^{1 / 2} \leq$ $1.42 \mathrm{GeV} . s_{i}^{1 / 2}$ is the energy above which one cannot consider that inelastic processes are negligible. The precise value $s_{i}^{1 / 2}$ where we separate "low energy" from "intermediate energy" depends on each wave.

In the low energy region we write model-independent parametrizations that take into account unitarity and analyticity; this last, by making a conformal mapping,

$$
s \rightarrow w(s)=\frac{\sqrt{s}-\sqrt{s_{i}-s}}{\sqrt{s}+\sqrt{s_{i}-s}}
$$

and expanding the function ${ }^{1} \cot \delta_{l}^{(I)}(s)$ in powers of this variable $w$. The details may be found in Appendix C, where we also explain how using this variable does not imply any model, while accelerating the convergence: we, generally speaking, only need between two and four terms in this expansion. We then fit experimental $\pi \pi$ phase shift data $[4,6]$ and, for the S0 wave at intermediate energy, also $\pi \pi \rightarrow \bar{K} K$ data [7]. In four cases (the waves D0, D2, F, G0) we include in the fit the values of the scattering length and, for the D0 wave, also the effective range parameter. These are obtained from experiment via the FroissartGribov representation; see Refs. [1,2] for details. In the case of the $\mathrm{P}$ wave, we do not fit the data on $\pi \pi$ scattering, but the vector form factor of the pion, which gives much more precise results [8]. In the intermediate energy region we make phenomenological fits to experimental phase shifts and elasticity parameters, basically polynomial fits. There is, however, an exception to this: the S0 wave. Here, most of the inelasticity is due to the $\bar{K} K$ channel, so we can make a two-channel calculation; see Ref. [2]. The precision of the fits, particularly for the S0, P, and D0 waves, is such that we require a (slight) improvement of the analyses of Refs. [1,2] in some cases.

\section{A. The S0 wave}

The first case where we improve on the analysis of Refs. [1,2] is the S0 wave, at low energy. Although this is discussed in detail in Ref. [3], we say a few words here for completeness. To write the corresponding expansion, it is necessary to separate off the pole of the effective range function that lies on the real axis, viz., the pole due to the so-called Adler zero of the partial wave amplitude. This lies near the beginning of the left hand cut, at $s=\frac{1}{2} z_{0}^{2}$, $z_{0} \simeq M_{\pi}$. We fit using the expression

\footnotetext{
${ }^{1}$ Up to a kinematical factor, and up to poles, that we separate explicitly; see the specific formulas for each wave.
} 


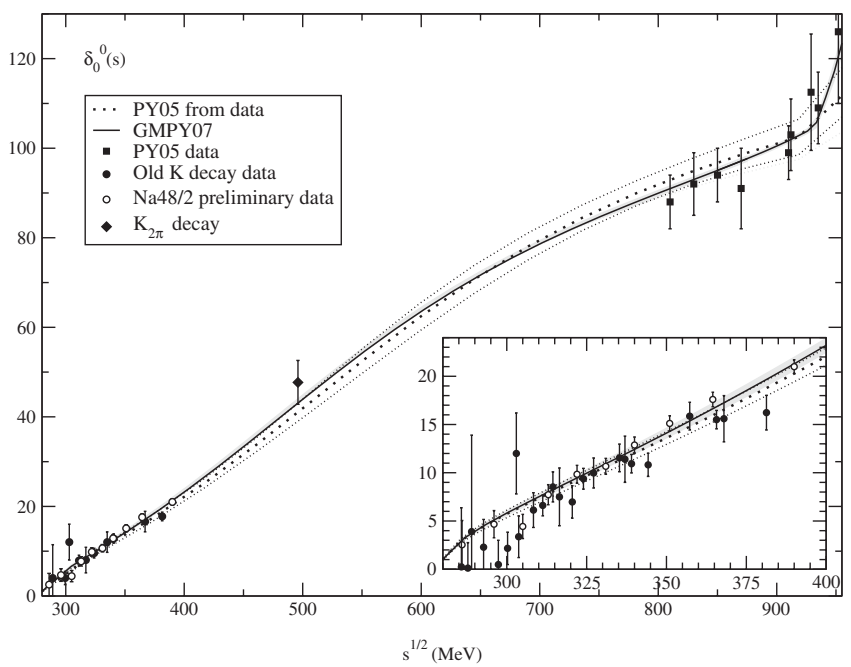

FIG. 1. The fits to S0 from Ref. [3] (solid line and gray band) and in Ref. [1] (dotted lines). In the inset, a blow-up of the low energy region. The fitted data from Refs. [4,6] are also shown.

$$
\begin{aligned}
\cot \delta_{0}^{(0)}(s)= & \frac{s^{1 / 2}}{2 k} \frac{M_{\pi}^{2}}{s-\frac{1}{2} z_{0}^{2}}\left\{\frac{z_{0}^{2}}{M_{\pi} \sqrt{s}}+B_{0}+B_{1} w(s)\right. \\
& \left.+B_{2} w(s)^{2}\right\}
\end{aligned}
$$

We here fix $z_{0}=M_{\pi}$; in Sec. V we will allow $z_{0}$ to vary. We find, taking the best fit $^{2}$ in [3],

$$
\begin{gathered}
B_{0}=4.3 \pm 0.3, \quad B_{1}=-26.9 \pm 0.6, \\
B_{2}=-14.1 \pm 1.4, \\
w(s)=\frac{\sqrt{s}-\sqrt{s_{0}-s}}{\sqrt{s}+\sqrt{s_{0}}-s}, \quad s_{0}=4 m_{K}^{2} .
\end{gathered}
$$

The ensuing numerical results for the phase shift are very similar to what was obtained in Ref. [1], but have a sounder theoretical basis and, above all, are much more accurate. The curves corresponding to this, and that in Ref. [1], are shown in Fig. 1, together with some experimental data. Because at $932 \mathrm{MeV}$ we match the low energy to the K-matrix fit at intermediate energy, and the low energy fit has changed (in particular with much smaller errors) from what we had in Ref. [2], we have also to slightly modify the parameters of the K-matrix fit. The resulting values for the parameters are given in

\footnotetext{
${ }^{2}$ In Ref. [3] we gave several different parametrizations for the S0 wave. These different parameters do not correspond to different physical scenarios, but just to the use of two or three terms in the conformal expansion, to what data sets are fitted, or to whether or not we explicitly factorize the zeros in the amplitudes. Such parametrizations correspond to the same physical scenario, as indeed their resulting phase shifts overlap within errors. We here choose, among those parametrizations, the one with better analytic properties, and which fits best the largest sample of consistent and reliable data points in the elastic region.
}

Appendix A. They do not change much from what we had in Ref. [2], but they are now determined more accurately.

The corrections due to isospin breaking have been computed for this wave, at very low energy; we discuss the (slight) modification they imply in Sec. VII, and the corresponding values of the parameters are given also in Appendix A.

\section{B. The S2 wave}

The second case where we improve on the analysis in Ref. [1,2] is for the S2 wave. The reason is that the precision of our calculations has improved so much that one is sensitive to the derivative of the phase shift as one crosses into the region where inelasticity is not negligible. In the case of the $\mathrm{S} 2$ wave, the lowest inelastic process is $\pi \pi \rightarrow \pi \pi \rho$, which is a (quasi-)three body process. Therefore, we expect $\delta_{0}^{(2)}(s)$ to be continuous, and with a continuous derivative, until the first two-body channel $(\rho \rho)$ opens. We take this into account now by altering the Ref. [1] fits to this wave, as follows. In the low energy region, $s^{1 / 2} \leq 932 \mathrm{MeV}$, we maintain the fit in Ref. [1]. We write

$$
\begin{gathered}
\cot \delta_{0}^{(2)}(s)=\frac{s^{1 / 2} M_{\pi}^{2}}{2 k s-2 z_{2}^{2}}\left\{B_{0}+B_{1} \frac{\sqrt{s}-\sqrt{s_{l}-s}}{\sqrt{s}+\sqrt{s_{l}-s}}\right\}, \\
z_{2} \equiv M_{\pi}, \quad s_{l}^{1 / 2}=1.05,
\end{gathered}
$$

$s^{1 / 2} \leq 932 \mathrm{MeV}$. We fix $z_{2}=M_{\pi}$; later, in Sec. V, we will allow $z_{2}$ to vary. For the errors, and since the low and high energy pieces are very strongly correlated, we take this into account and get somewhat improved errors. Altogether we find a $\chi^{2} /$ d.o.f. $=11.2 /(21-2)$ and the parameters

$$
B_{0}=-80.4 \pm 2.8, \quad B_{1}=-73.6 \pm 10.5 \text {. }
$$

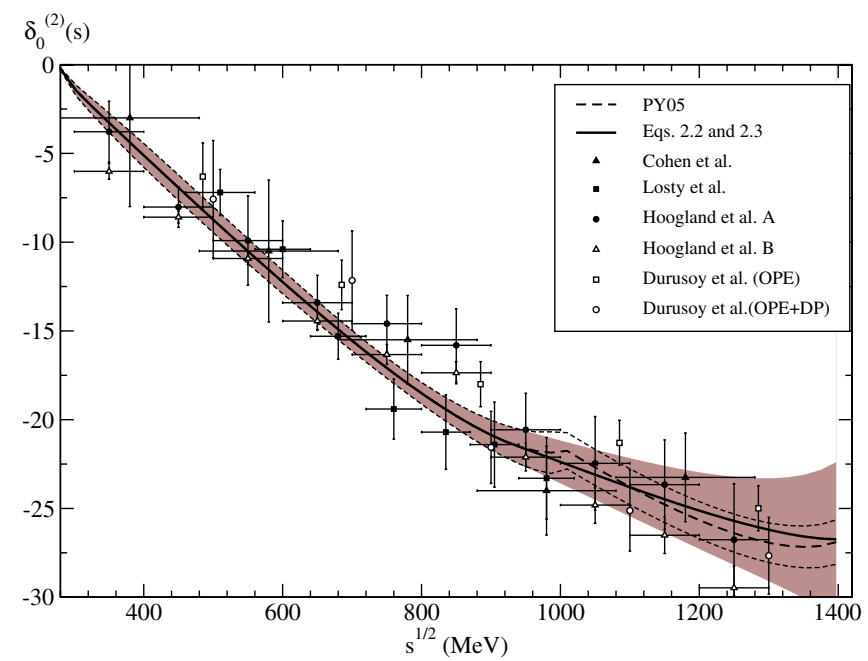

FIG. 2 (color online). The fits to the S2 phase shift here (solid line and gray band) and in Ref. [1] (dashed lines). Some experimental data from Ref. [6] are also shown. 
For the high energy region we neglect the inelasticity below $1.45 \mathrm{GeV}$ for the fit to the phase. We then fit high energy data $\left(s^{1 / 2} \geq 0.95 \mathrm{GeV}\right)$, requiring agreement of the central value and derivative with the low energy determination given in (2.2) at the energy $s_{M}^{1 / 2}=932 \mathrm{MeV}$. We write

$$
\begin{aligned}
\cot \delta_{0}^{(2)}(s)= & \frac{s^{1 / 2} M_{\pi}^{2}}{2 k s-2 M_{\pi}^{2}}\left\{B_{h 0}+B_{h 1}\left[w_{h}(s)-w_{h}\left(s_{M}\right)\right]\right. \\
& \left.+B_{h 2}\left[w_{h}(s)-w_{h}\left(s_{M}\right)\right]^{2}\right\}, \\
s^{1 / 2} \geq & 932 \mathrm{MeV}, \quad B_{h 0}=B_{0}+B_{1} w_{l}\left(s_{M}\right) \\
B_{h 1}= & \left.B_{1} \frac{\partial w_{l}(s)}{\partial w_{h}(s)}\right|_{s=s_{M}},
\end{aligned}
$$

and

$$
\begin{array}{ll}
w_{l}(s)=\frac{\sqrt{s}-\sqrt{s_{l}-s}}{\sqrt{s}+\sqrt{s_{l}-s}}, & s_{l}^{1 / 2}=1050, \\
w_{h}(s)=\frac{\sqrt{s}-\sqrt{s_{h}-s}}{\sqrt{s}+\sqrt{s_{h}-s}}, & s_{h}^{1 / 2}=1450 .
\end{array}
$$

$B_{h 2}$ is a free parameter. We get a reasonable $\chi^{2} /$ d.o.f. $=$ $13.8 /(13-1)$ and

$$
B_{h 2}=112 \pm 38
$$

Both the present fit and that in Ref. [1] may be found in Fig. 2.

The present fit has slightly smaller errors than the old fit in Ref. [1] in the low energy region, and larger ones above $1 \mathrm{GeV}$.

The inelasticity we still describe by the empirical fit in Ref. [1]:

$$
\eta_{0}^{(2)}(s)= \begin{cases}1-\epsilon\left(1-s_{l} / s\right)^{3 / 2}, & \epsilon=0.18 \pm 0.12, \quad s>s_{l}=(1.05 \mathrm{GeV})^{2} \\ 1, & s<s_{l}=(1.05 \mathrm{GeV})^{2}\end{cases}
$$

\section{The inelasticity for the Do wave}

We now consider the elasticity parameter for the D0 wave. In Ref. [1,2] we made a phenomenological fit to $\eta_{2}^{(0)}$ :

$$
\eta_{2}^{(0)}(s)= \begin{cases}1, & s<4 m_{K}^{2}, \\ 1-\epsilon \frac{k_{2}(s)}{k_{2}\left(M_{f_{2}}^{2}\right)}, & s>4 m_{K}^{2}, \quad \epsilon=0.262 \pm 0.030, \quad k_{2}=\sqrt{s / 4-m_{K}^{2}} .\end{cases}
$$

This provides a fit to the elasticity parameter on the average.

The problem with (2.5) is that it rises too brusquely at the $\bar{K} K$ threshold, ${ }^{3}$ proportional to $k_{2}$, which causes distortions for $s^{1 / 2}$ near $2 m_{K}$; a behavior $k_{2}^{5}$ near this threshold would be indicated. For this reason, we here also try the following parametrization:

$$
\begin{aligned}
& \eta_{2}^{(0)}(s)=\left\{\begin{array}{l}
1, \quad s<4 m_{K}^{2}, \\
1-\epsilon\left(1-\frac{4 m_{K}^{2}}{s}\right)^{5 / 2}\left(1-\frac{4 m_{K}^{2}}{M_{f_{2}}^{2}}\right)^{-5 / 2}\left\{1+r\left[1-\frac{k_{2}(s)}{k_{2}\left(M_{f_{2}}^{2}\right)}\right]\right\}, \\
s>4 m_{K}^{2}, \quad k_{2}=\sqrt{\frac{s}{4}-m_{K}^{2}} .
\end{array}\right. \\
& \epsilon=0.284 \pm 0.030, \quad r=2.54 \pm 0.31, \quad M_{f_{2}}=1275.4 \mathrm{MeV} .
\end{aligned}
$$

This will underestimate the inelasticity near $2 m_{K}$, since, in fact, most of the inelasticity of the D0 wave is not due to $\bar{K} K$ but to the four pion states; but will have a smooth threshold behavior. Moreover, (2.6) is more flexible than (2.5) and it will, by construction, have the correct inelasticity around the $f_{2}(1270)$ resonance, which is the most

\footnotetext{
${ }^{3}$ This type of problem is important for the D0 wave, because the $\bar{K} K$ threshold is not far from the very strong $f_{2}(1270)$ resonance; but it also exists for $\mathrm{P}$ and $\mathrm{D} 2$ waves. We have checked that, in the case of these waves, the influence of the incorrect threshold behavior is much smaller than our errors, hence negligible.
}

important region. If evaluating the dispersion relations with (2.5) and (2.6) we find that they are almost equivalent. We will use (2.6) in our calculations in the present paper because, as stated, it reproduces best the inelasticity around the $f_{2}(1270)$ resonance.

The elasticity parameter here and that from Ref. [1] are shown in Fig. 3.

\section{The D2 wave over the whole range}

Finally, we discuss the D2 wave. The data for the corresponding phase shift are very poor (and nonexistent for the inelasticity). For this reason, we made, in Ref. [1], a 


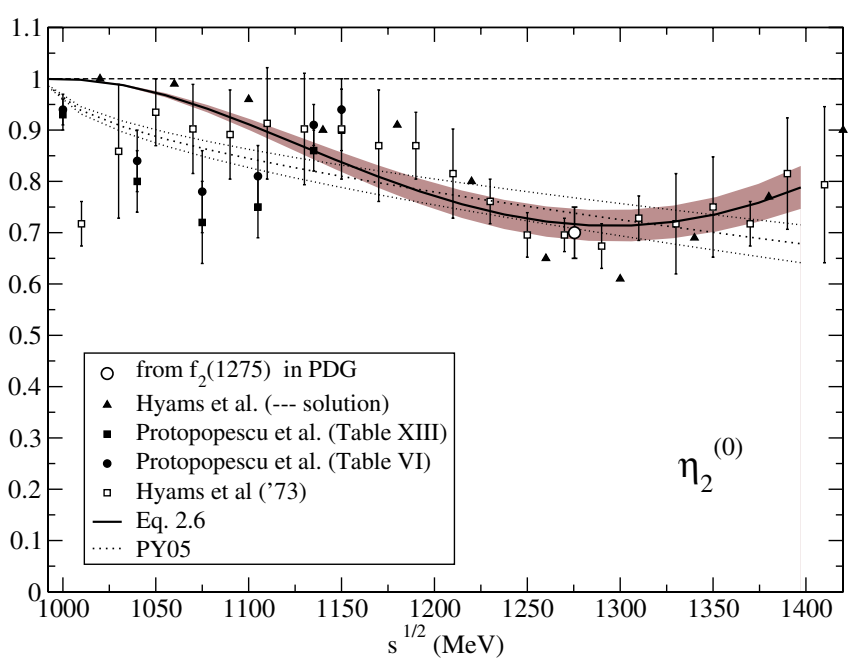

FIG. 3 (color online). Fit to $\eta_{2}^{(0)}$ (continuous line and dark area that covers the uncertainty), from Eq. (2.6) here. The dotted lines follow from Ref. [2] [Eq. (2.5) here]. The elasticity on the $f_{2}(1270)$, from the Particle Data Tables, is also shown as a large white dot; other data are from Ref. [6].

phenomenological fit in the whole energy range from threshold to $1.42 \mathrm{GeV}$. We wrote

$$
\begin{aligned}
\cot \delta_{2}^{(2)}(s)= & \frac{s^{1 / 2}}{2 k^{5}}\left\{B_{0}+B_{1} w(s)+B_{2} w(s)^{2}\right\} \\
& \times \frac{M_{\pi}^{4} s}{4\left(M_{\pi}^{2}+\Delta^{2}\right)-s}
\end{aligned}
$$

with $\Delta$ a free parameter fixing the zero of the phase shift and

$$
w(s)=\frac{\sqrt{s}-\sqrt{s_{0}-s}}{\sqrt{s}+\sqrt{s_{0}-s}}, \quad s_{0}^{1 / 2}=1450 \mathrm{MeV},
$$

and we got a mediocre fit, $\chi^{2} /$ d.o.f. $=71 /(25-4)$; the values of the parameters were

$$
\begin{array}{ll}
B_{0}=(2.4 \pm 0.3) \times 10^{3}, & B_{1}=(7.8 \pm 0.8) \times 10^{3}, \\
B_{2}=(23.7 \pm 3.8) \times 10^{3}, & \Delta=196 \pm 20 \mathrm{MeV} .
\end{array}
$$

When we fitted requiring fulfillment of dispersion relations, the parameters moved to (Ref. [1], Appendix A)

$$
\begin{array}{ll}
B_{0}=(2.9 \pm 0.2) \times 10^{3}, & B_{1}=(7.3 \pm 0.8) \times 10^{3}, \\
B_{2}=(25.4 \pm 3.6) \times 10^{3}, & \Delta=212 \pm 19 \mathrm{MeV} .
\end{array}
$$

The corresponding phase shift moves by a little more than 1 standard deviation. This shows that the errors in (2.7b) were underestimated. We improve this by keeping the central values of the parameters we found in the fit to data, but enlarging the errors by adding quadratically the difference between the central values in (2.7a) and (2.7b), which may be considered as a "systematic" error in the fit. ${ }^{4}$ Thus we find the parameters

$$
\begin{array}{ll}
B_{0}=(2.4 \pm 0.5) \times 10^{3}, & B_{1}=(7.8 \pm 1.0) \times 10^{3}, \\
B_{2}=(23.7 \pm 4.2) \times 10^{3}, & \Delta=196 \pm 25 \mathrm{MeV} .
\end{array}
$$

It should be noted that the errors in $\delta_{2}^{(2)}$ are still small, compared to the experimental errors; (2.7d) gives errors for $\delta_{2}^{(2)}$ below the level of $0.8^{\circ}$.

For the elasticity parameter we write the same formula as in Ref. [2]: above $1.05 \mathrm{GeV}$,

$$
\begin{gathered}
\eta_{2}^{(2)}(s)=1-\epsilon(1-\hat{s} / s)^{3}, \quad \hat{s}^{1 / 2}=1.05 \mathrm{GeV}, \\
\epsilon=0.2 \pm 0.2 .
\end{gathered}
$$

\section{E. The G0 wave}

With respect to the G0 wave, we gave in Ref. [1] a parametrization for its imaginary part based on dominance by the resonance $f_{4}$,

$$
\begin{aligned}
\operatorname{Im}_{4}^{(0)}(s)= & \left(\frac{k(s)}{k\left(M_{f_{4}}^{2}\right)}\right)^{18} \\
& \times \mathrm{BR} \frac{M_{f_{4}}^{2} \Gamma^{2}}{\left(s-M_{f_{4}}^{2}\right)^{2}+M_{f_{4}}^{2} \Gamma^{2}\left[k(s) / k\left(M_{f_{4}}^{2}\right)\right]^{18}}, \\
s^{1 / 2} \geq & 1 \mathrm{GeV}, \quad \mathrm{BR}=0.17 \pm 0.02, \\
M_{f_{4}}= & 2025 \pm 8 \mathrm{MeV}, \quad \Gamma=194 \pm 13 \mathrm{MeV} .
\end{aligned}
$$

Unfortunately, this much underestimates the value at low energy. In fact, from the Froissart-Gribov representation we can evaluate rather accurately the scattering length, finding $a_{4}^{(0)}=(8.0 \pm 0.4) \times 10^{-6} M_{\pi}^{-9}$. At low energy one can write

$$
\operatorname{Im} \hat{f}_{4}^{(0)}(s) \simeq[k(s)]^{18}\left[a_{4}^{(0)}\right]^{2},
$$

which disagrees with what one would find from (2.8) by many orders of magnitude. A simple formula that interpolates between low and high energy is

\footnotetext{
${ }^{4}$ It should be remarked that the present method, as indeed any method for estimating systematic errors, is arbitrary to a large extent. In Ref. [1] we took into account the poor quality of the fit (which reflects the incompatibility of the various sets of experimental data) by scaling the purely statistical errors by the square root of the $\chi^{2} /$ d.o.f., $\sqrt{71 /(25-4)} \simeq 1$. . This produced $(2.7 \mathrm{~b})$. We could have scaled instead by the $\chi^{2} /$ d.o.f. itself; this would have given errors like in $(2.7 \mathrm{~d})$ for the parameter, $B_{0}$, and a bit larger than those in (2.7d) for the other parameters. Likewise, we could have taken as central values the averages of the central values in (2.7b) and (2.7c). We consider our method to be reasonable and, as we find in the present paper, the increase in the errors quite justified.
} 


$$
\begin{aligned}
\operatorname{Im} \hat{f}_{4}^{(0)}(s)= & \left(\frac{k(s)}{k\left(M_{f_{4}}^{2}\right)}\right)^{18} \\
& \times \mathrm{BR} \frac{M_{f_{4}}^{2} \Gamma^{2} \mathrm{e}^{2 c\left(1-s / M_{f_{4}}^{2}\right)^{2}}}{\left(s-M_{f_{4}}^{2}\right)^{2}+M_{f_{4}}^{2} \Gamma^{2}\left[k(s) / k\left(M_{f_{4}}^{2}\right)\right]^{18}}, \\
\mathrm{BR}= & 0.17 \pm 0.02, \quad M_{f_{4}}=2025 \pm 8 \mathrm{MeV}, \\
\Gamma= & 194 \pm 13 \mathrm{MeV}, \quad c=9.23 \pm 0.46 .
\end{aligned}
$$

This gives the correct values near the $f_{4}$ resonance and at low energy, the only regions where we have experimental information. Note that this interpolation is rather arbitrary, but there is no point in trying to improve it as there are no data to which one can fit more realistic formulas. Any reasonable interpolation would give the same order of magnitude estimate for the contribution of this wave to dispersion relations and sum rules.

We have, in our calculations, not taken into account the contributions of G0 or G2 waves other than to check that they are considerably smaller than the experimental errors: this is the only interest of the parametrization.

\section{F. Low energy S0, P, and F waves: Ghost removal}

When cutting the low energy expansions

$$
\cot \delta(s)=K(s)\left\{B_{0}+B_{1} w+\cdots\right\}
$$

(with $K$ an appropriate kinematical factor) at a finite order, a ghost, i.e., a spurious pole in the partial wave amplitude $\hat{f}(s)$ appears in the vicinity of the point $s=0$ for the S0, P, and $\mathrm{F}$ waves. As remarked in Ref. [3] (see especially Appendix A there), such ghost poles are rather harmless, their effect being at the percent level: removing the ghosts is little more than an aesthetical requirement. Nevertheless, we will here improve our formulas by writing them in such a manner that the ghosts disappear. This was already done for the S0 wave in Ref. [3], and the S0 parametrization used in the present paper takes this into account; now we remove also the ghosts for $\mathrm{P}$ and $\mathrm{F}$ waves. As a matter of fact, this improves the consistency of our results, slightly but systematically.

In Refs. $[1,2]$ we used the following formula for the $\mathrm{P}$ wave:

$$
\begin{aligned}
\cot \delta_{1}(s) & =\frac{s^{1 / 2}}{2 k^{3}}\left(M_{\rho}^{2}-s\right)\left\{B_{0}+B_{1} \frac{\sqrt{s}-\sqrt{s_{0}-s}}{\sqrt{s}+\sqrt{s_{0}-s}}\right\}, \\
s_{0}^{1 / 2} & =1.05 \mathrm{GeV} .
\end{aligned}
$$

The best result, from Ref. [8] (see also Ref. [1]), is

$$
\begin{gathered}
B_{0}=1.069 \pm 0.011, \quad B_{1}=0.13 \pm 0.05, \\
M_{\rho}=773.6 \pm 0.9 \mathrm{MeV} .
\end{gathered}
$$

Instead of this we now write a parametrization where the ghost is absent,

$$
\begin{aligned}
\cot \delta_{1}(s) & =\frac{s^{1 / 2}}{2 k^{3}}\left(M_{\rho}^{2}-s\right)\left\{\frac{2 M_{\pi}^{3}}{M_{\rho}^{2} \sqrt{s}}+B_{0}+B_{1} \frac{\sqrt{s}-\sqrt{s_{0}-s}}{\sqrt{s}+\sqrt{s_{0}-s}}\right\}, \\
s_{0}^{1 / 2} & =1.05 \mathrm{GeV},
\end{aligned}
$$

and find

$$
B_{0}=1.055 \pm 0.011, \quad B_{1}=0.15 \pm 0.05 ;
$$

the difference with what follows from (2.11) is less than $0.7 \%$ at the $\pi \pi$ threshold, decreasing to $0.05 \%$ at the $\bar{K} K$ threshold.

For the $\mathrm{F}$ wave, one can remove the ghost without changing the parameters we found in Ref. [1] (within the significant digits), so we have

$$
\begin{gathered}
\cot \delta_{3}(s)=\frac{s^{1 / 2}}{2 k^{7}} M_{\pi}^{6}\left\{\frac{2 \lambda M_{\pi}}{\sqrt{s}}+B_{0}+B_{1} \frac{\sqrt{s}-\sqrt{s_{0}-s}}{\sqrt{s}+\sqrt{s_{0}-s}}\right\} \\
s_{0}^{1 / 2}=1.45 \mathrm{GeV}, \quad B_{0}=(1.09 \pm 0.03) \times 10^{5} \\
B_{1}=(1.41 \pm 0.04) \times 10^{5}, \quad \lambda=0.051 \times 10^{5} .
\end{gathered}
$$

\section{G. Other Waves}

The results of the remaining fits for this UFD set may be found in Refs. [1,2], with details of the fitting procedure and the far from trivial matter of the selection of data. The results of all the fits are collected in Appendix A, for ease of reference.

\section{H. Matching}

Before turning to the calculations of forward dispersion relations, and Roy equations, a few words have to be said about the matching points between our low energy and intermediate energy regimes, and between intermediate energy and high energy regimes, that we discuss later. Such matchings are, of course, artificial:, for example, and as discussed about the D0 wave, the inelasticity is small, but not zero, below the $\bar{K} K$ threshold. A fully satisfactory matching is however not possible; it would require a multichannel evaluation, and hence introducing a number of parameters impossible to fix with the existing experimental data. In Ref. [2], and here, we have requested matching of the central values of the phase shifts, $\delta_{l}^{(I)}(s)$, at the matching points themselves, usually (but not always) the $\bar{K} K$ threshold. However, and because the errors in the low energy and intermediate energy ranges are independent, this produces jumps (when varying the parameters inside their error bars) which increase the errors of the dispersive integrals artificially. This is, unfortunately, an unavoidable feature of our analysis: the results deteriorate somewhat when one is very near the matching points.

In fact, the situation is even less clear near the $\bar{K} K$ threshold. In our analysis we neglect isospin breaking effects, and therefore we have taken it at an average 
between the $K^{+} K^{-}$and $\bar{K}^{0} K^{0}$ thresholds, $s^{1 / 2}=$ $992 \mathrm{MeV}$. Since the $K^{+} K^{-}$and $\bar{K}^{0} K^{0}$ thresholds differ by some $8 \mathrm{MeV}$, the threshold itself is thus not well defined to this extent: $992 \pm 4 \mathrm{MeV}$. All in all, the net result is that our dispersive (or even direct calculations) of the $\pi \pi$ amplitudes suffer from uncontrollable errors in a, fortunately narrow, band of less than or around $6 \mathrm{MeV}$ (to be on the safe side) around the matching points. We have avoided these matching regions when calculating the fulfillment of dispersive relations.

With respect to the matching between intermediate and high energy regions, the situation is different. It is clear that, near $s^{1 / 2}=1420 \mathrm{MeV}$, which is the corresponding matching point, the Regge expression can only agree with the real amplitudes in the mean (as can be seen in the cases where we have precise data, as for $\pi N$ scattering). We expect that, since these Regge amplitudes only appear in integrals, the fluctuations will be averaged out to lie within the errors. However, we here have a problem similar to that of the low energy matching with intermediate energy: this lack of correlation of the errors causes artificially enhanced error bars near the matching points. Therefore, our calculation should be used excluding a (narrow) band below the matching point. Here we have refrained to compare calculations above $1400 \mathrm{MeV}$, which is sufficient to render most of the fluctuations smaller than the experimental errors.

\section{REGGE FORMULAS}

Regge formulas have been obtained for $\pi \pi$ scattering, in the forward direction, by fitting experimental data for the various $\pi \pi$ total cross sections. This provides expressions that are not very precise. One improves this by use of factorization. It is then possible to include information on total cross sections for $\pi N$ and $N N$ scattering [9] ( $N N$ includes antinucleon-nucleon scattering), which furnishes us with precise results for the contributions to $\pi \pi$ scattering of the three Regge poles ${ }^{5} P, P^{\prime}$, and $\rho$. Here, we will use these Regge expressions above $1.42 \mathrm{GeV}$.

These results are sufficient to calculate forward dispersion relations. For Roy equations, however, we require also the imaginary parts of the scattering amplitudes, $\operatorname{Im} F(s, t)$, for rather large values of $|t|$; in our calculation, up to $t=$ $-0.43 \mathrm{GeV}^{2}$. In fact, these values are so large that one does not expect Regge theory to hold in the extreme range. What we do to circumvent this problem is to enlarge the errors in the $t$ dependence of the parametrizations so that they cover, in the whole $t$ range, all fits to experimental data. This will provide, at large $t$, a phenomenological representation of the corresponding scattering amplitude. For isospin zero exchange, we take the expressions given in Ref. [1], in the forward direction, and enlarge the errors away from the forward direction. For $\rho$ exchange we take,

\footnotetext{
${ }^{5}$ The $P^{\prime}$ is in fact a combination of two Regge trajectories, associated with the $f_{2}(1270)$ and $f_{2}^{\prime}(1525)$ resonances.
}

at $t=0$, the parameters described in Ref. [2] and, for $t \neq$ 0 , an uncertainty that covers the extreme fits in Ref. [10] for $\pi N$ scattering, and assume that $\pi \pi$ scattering will vary in a similar manner.

We write

$$
\begin{aligned}
& \operatorname{Im} F^{\left(I_{t}=1\right)}(s, t) \underset{\substack{s \rightarrow \infty \\
\text { tfixed }}}{\simeq} \beta_{\rho} \frac{1+\alpha_{\rho}(t)}{1+\alpha_{\rho}(0)} \varphi(t) \mathrm{e}^{b t}(s / \hat{s})^{\alpha_{\rho}(t)} \\
& \alpha_{\rho}(t)=\alpha_{\rho}(0)+t \alpha_{\rho}^{\prime}+\frac{1}{2} t^{2} \alpha_{\rho}^{\prime \prime}, \\
& \beta_{\rho}=1.22 \pm 0.14 \\
& \alpha_{\rho}(0)=0.46 \pm 0.02 \\
& \alpha_{\rho}^{\prime}=0.90 \mathrm{GeV}^{-2} \\
& \alpha_{\rho}^{\prime \prime}=-0.3 \mathrm{GeV}^{-4} \\
& \varphi(t)=1+d_{\rho} t+e_{\rho} t^{2} \\
& b=2.4 \pm 0.2 \mathrm{GeV}^{-2}
\end{aligned}
$$

$\hat{s}$ is a scale parameter, that we consistently take $\hat{s} \equiv$ $1 \mathrm{GeV}^{2}$. We set

$$
d_{\rho}=2.4 \pm 0.5, \quad e_{\rho}=0 \pm 2.5 \mathrm{GeV}^{-4} .
$$

For the Pomeron and $P^{\prime}$, one can write, also for $s^{1 / 2}>$ $1.42 \mathrm{GeV}$,

$$
\begin{aligned}
& \operatorname{Im} F_{\pi \pi}^{\left(I_{t}=0\right)}(s, t) \simeq_{\substack{s \rightarrow \infty \\
\text { tfixed }}} P(s, t)+P^{\prime}(s, t), \\
& P(s, t)=\beta_{P} \Psi_{P}(t) \alpha_{P}(t) \frac{1+\alpha_{P}(t)}{2} \mathrm{e}^{b t}(s / \hat{s})^{\alpha_{P}(t)}, \\
& \alpha_{P}(t)=1+t \alpha_{P}^{\prime}, \quad \beta_{P}=2.54 \pm 0.04, \\
& \alpha_{P}^{\prime}=0.20 \pm 0.10 \mathrm{GeV}^{-2}, \quad \Psi_{P}(t)=1+c_{P} t, \\
& P^{\prime}(s, t)=\beta_{P^{\prime}} \Psi_{P^{\prime}}(t) \frac{\alpha_{P^{\prime}}(t)\left[1+\alpha_{P^{\prime}}(t)\right]}{\alpha_{P^{\prime}}(0)\left[1+\alpha_{P^{\prime}}(0)\right]} \mathrm{e}^{b t}(s / \hat{s})^{\alpha_{P^{\prime}}(t),}, \\
& \alpha_{P^{\prime}}(t)=\alpha_{P^{\prime}}(0)+t \alpha_{P^{\prime}}^{\prime}, \quad \beta_{P^{\prime}}=0.83 \pm 0.05, \\
& \alpha_{P^{\prime}}(0)=0.54 \pm 0.02, \quad \alpha_{P^{\prime}}^{\prime}=0.90 \mathrm{GeV}^{-2}, \\
& \Psi_{P^{\prime}}(t)=1+c_{P^{\prime}} t, \quad b=2.4 \pm 0.2 \mathrm{GeV}^{-2} .
\end{aligned}
$$

We may fix

$$
c_{P}=0.0 \pm 1.0 \mathrm{GeV}^{-2}, \quad c_{P^{\prime}}=-0.4 \pm 0.4 \mathrm{GeV}^{-2} \text {. }
$$

If we do so, we cover the fits of Rarita et al. [10] and of Froggatt and Petersen [11].

Note that we do not give errors for the slopes $\alpha_{\rho}^{\prime}$ and $\alpha_{P^{\prime}}^{\prime}$ because the variation of $\varphi, \Psi_{P^{\prime}}$ covers possible variations of the Regge slopes: $\varphi$ varies a lot at large $t$. In fact, we have a range of variation

$$
-0.56 \lesssim \varphi\left(-0.4 \mathrm{GeV}^{2}\right) \lesssim 0.64,
$$


and something similar for $\Psi_{P^{\prime}}$. Fortunately, however, the evaluations for the Roy equations below $1 \mathrm{GeV}$ depend very little on the scattering amplitudes for large $s$ and large $|t|$.

Finally, for exchange of isospin two we write

$$
\begin{aligned}
& \operatorname{Im} F^{\left(I_{t}=2\right)}(s, t) \underset{s \rightarrow \infty}{\simeq} \beta_{2} \mathrm{e}^{b t}(s / \hat{s})^{\alpha_{\rho}(t)+\alpha_{\rho}(0)-1}, \\
& \beta_{2}=0.2 \pm 0.2, \quad s \geq(1.42 \mathrm{GeV})^{2}
\end{aligned}
$$

These fits are expected to represent experimental data for energies between $1.42 \mathrm{GeV}$ and $\sim 20 \mathrm{GeV}$ and for $4 M_{\pi}^{2} \geq t \geq-0.4 \mathrm{GeV}^{2}$, with less reliability at the more negative values of $t$. At values of $s^{1 / 2}$ larger than $20 \mathrm{GeV}$, one would have to use more complicated formulas, taking into account, in particular, the logarithmic growth of the total cross sections [9]. For our purposes the formulas given above are sufficiently accurate, since the influence of the energy region much above $20 \mathrm{GeV}$ for forward dispersion relations or Roy equations is negligible.

The values of the Regge parameters can be improved by requiring verification of dispersion relations, and of two sum rules that relate directly the Regge behavior, for nonzero $t$, to low energy amplitudes [see below Eqs. (5.1) and (5.2)]. The resulting numbers are collected in Appendix B.

\section{DISPERSION RELATIONS}

\section{A. Forward dispersion relations}

In this section we will evaluate forward dispersion relations for the three independent $\pi \pi$ scattering amplitudes. For these calculations we will take the parameters for all partial waves from the fits to data described in the previous sections (and collected in Appendix A).

Although the form of the dispersion relations has been given in Ref. [1], we repeat them here. For $\pi^{0} \pi^{0}$ scattering we write

$$
\begin{aligned}
& \operatorname{Re} F_{00}(s)-F_{00}\left(4 M_{\pi}^{2}\right) \\
& =\frac{s\left(s-4 M_{\pi}^{2}\right)}{\pi} \text { P.P. } \int_{4 M_{\pi}^{2}}^{\infty} \mathrm{d} s^{\prime} \\
& \quad \times \frac{\left(2 s^{\prime}-4 M_{\pi}^{2}\right) \operatorname{Im} F_{00}\left(s^{\prime}\right)}{s^{\prime}\left(s^{\prime}-s\right)\left(s^{\prime}-4 M_{\pi}^{2}\right)\left(s^{\prime}+s-4 M_{\pi}^{2}\right)} .
\end{aligned}
$$

The result of the calculation is shown in Fig. 4(a), where the continuous curve is the real part evaluated from the parametrizations, and the dashed line is the result of the dispersive integral, i.e., the right-hand side of (4.1a).

The dispersion relation for $\pi^{0} \pi^{+}$scattering reads, with $F_{0+}(s)$ the forward $\pi^{0} \pi^{+}$amplitude,
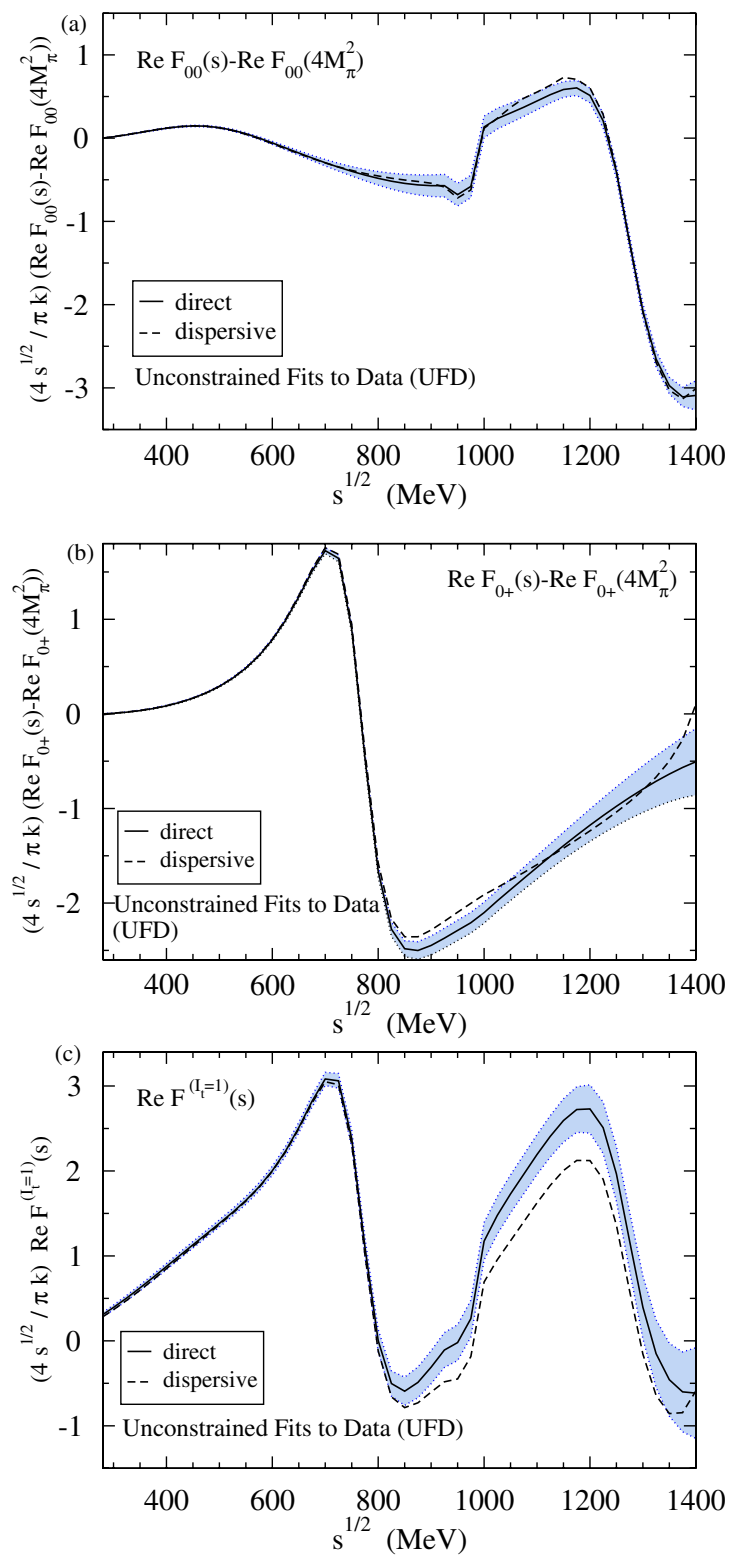

FIG. 4 (color online). Results for forward dispersion relations. Continuous lines: real part, evaluated directly with the UFD parametrizations. Dashed lines: the result of the dispersive integrals. The gray bands cover the uncertainties in the difference between both. From top to bottom: (a) the $\pi^{0} \pi^{0}$ FDR, (b) the $\pi^{0} \pi^{+}$FDR, (c) the FDR for $I_{t}=1$ scattering.

$$
\begin{aligned}
& \operatorname{Re} F_{0+}(s)-F_{0+}\left(4 M_{\pi}^{2}\right) \\
& =\frac{s\left(s-4 M_{\pi}^{2}\right)}{\pi} \text { P.P. } \int_{4 M_{\pi}^{2}}^{\infty} \mathrm{d} s^{\prime} \\
& \quad \times \frac{\left(2 s^{\prime}-4 M_{\pi}^{2}\right) \operatorname{Im} F_{0+}\left(s^{\prime}\right)}{s^{\prime}\left(s^{\prime}-s\right)\left(s^{\prime}-4 M_{\pi}^{2}\right)\left(s^{\prime}+s-4 M_{\pi}^{2}\right)} .
\end{aligned}
$$

In Fig. 4(b) we show the fulfillment of (4.1b).

Finally, the dispersion relation for the $I_{t}=1$ scattering amplitude does not require subtractions, and reads 


$$
\begin{aligned}
\operatorname{Re} F^{\left(I_{t}=1\right)}(s, 0)= & \frac{2 s-4 M_{\pi}^{2}}{\pi} \text { P.P. } \int_{4 M_{\pi}^{2}}^{\infty} \mathrm{d} s^{\prime} \\
& \times \frac{\operatorname{Im} F^{\left(I_{t}=1\right)}\left(s^{\prime}, 0\right)}{\left(s^{\prime}-s\right)\left(s^{\prime}+s-4 M_{\pi}^{2}\right)} .
\end{aligned}
$$

The result is shown graphically in Fig. 4(c).

To measure quantitatively the fulfillment of the dispersion relations we evaluate the average (squared) distance between the real parts, calculated with our parametrizations, and the same real parts but now calculated with the aid of the dispersive integrals, a quantity that we denote by $\bar{d}^{2}$. This quantity is defined as follows. First, we rewrite the dispersion relations as the discrepancies $\Delta_{i}$ (shown graphically in Fig. 5),

$$
\begin{aligned}
& \Delta_{00}(s) \equiv \operatorname{Re} F_{00}(s)-F_{00}\left(4 M_{\pi}^{2}\right)-\frac{s\left(s-4 M_{\pi}^{2}\right)}{\pi} \mathrm{P} . \mathrm{P} . \int_{4 M_{\pi}^{2}}^{\infty} \mathrm{d} s^{\prime} \frac{\left(2 s^{\prime}-4 M_{\pi}^{2}\right) \operatorname{Im} F_{00}\left(s^{\prime}\right)}{s^{\prime}\left(s^{\prime}-s\right)\left(s^{\prime}-4 M_{\pi}^{2}\right)\left(s^{\prime}+s-4 M_{\pi}^{2}\right)}, \\
& \Delta_{0+}(s) \equiv \operatorname{Re} F_{0+}(s)-F_{0+}\left(4 M_{\pi}^{2}\right)-\frac{s\left(s-4 M_{\pi}^{2}\right)}{\pi} \text { P.P. } \int_{4 M_{\pi}^{2}}^{\infty} \mathrm{d} s^{\prime} \frac{\left(2 s^{\prime}-4 M_{\pi}^{2}\right) \operatorname{Im} F_{0+}\left(s^{\prime}\right)}{s^{\prime}\left(s^{\prime}-s\right)\left(s^{\prime}-4 M_{\pi}^{2}\right)\left(s^{\prime}+s-4 M_{\pi}^{2}\right)},
\end{aligned}
$$

and

$$
\Delta_{1}(s) \equiv \operatorname{Re} F^{\left(I_{t}=1\right)}(s, 0)-\frac{2 s-4 M_{\pi}^{2}}{\pi} \text { P.P. } \int_{4 M_{\pi}^{2}}^{\infty} \mathrm{d} s^{\prime} \frac{\operatorname{Im} F^{\left(I_{t}=1\right)}\left(s^{\prime}, 0\right)}{\left(s^{\prime}-s\right)\left(s^{\prime}+s-4 M_{\pi}^{2}\right)}
$$

These quantities would vanish, $\Delta_{i}=0$, if the dispersion relations were exactly satisfied. Because our fits have errors, we can only require vanishing within the uncertainties induced in the $\Delta_{i}$, that we call $\delta \Delta_{i}$. Therefore, we define the quantities (average discrepancies)

$$
\bar{d}_{i}^{2} \equiv \frac{1}{\text { number of points }} \sum_{n}\left(\frac{\Delta_{i}\left(s_{n}\right)}{\delta \Delta_{i}\left(s_{n}\right)}\right)^{2} .
$$

This we do for all three relations (4.2). The values of the $s_{n}$ are taken at energy intervals of $25 \mathrm{MeV}$. For the dispersion relation for $I_{t}=1$, we also include the value at threshold, known at times as the (first) Olsson sum rule. For the other two dispersion relations, since the $\Delta$ vanish identically at threshold, we include a point below threshold, at $s=2 M_{\pi}^{2}$. This is useful, among other things, to fix the location of the Adler zeros for the S0, S2 waves.

If we had a fit to FDRs (which we do not) instead of an evaluation, $\bar{d}^{2}$ would be the average chi-squared of the fit: in our case, $\bar{d}^{2}$ is simply a measure of how well the forward dispersion relations are satisfied by the data fits, which are independent for each wave, and independent of dispersion relations. When calculating this $\bar{d}^{2}$, we use, in the present section, the parameters for phase shifts and inelasticities discussed in the previous sections, and collected in Appendix A.

The average discrepancies in the various cases are given in Eq. (4.4) below:

$$
\begin{array}{ccc} 
& s^{1 / 2} \leq 932 \mathrm{MeV} & s^{1 / 2} \leq 1420 \mathrm{MeV} \\
\pi^{0} \pi^{0} \text { FDR } & \bar{d}^{2}=0.12 & \bar{d}^{2}=0.29 \\
\pi^{0} \pi^{+} \text {FDR } & \bar{d}^{2}=0.84 & \bar{d}^{2}=0.86 \\
I_{t}=1 \mathrm{FDR} & \bar{d}^{2}=0.66 & \bar{d}^{2}=1.87 .
\end{array}
$$

Below $932 \mathrm{MeV}$, the average $\bar{d}^{2}$ shows a remarkably good fulfillment of FDRs. Still, the situation over the whole range is such that the $\bar{d}^{2}$ for the $I_{t}=1 \mathrm{FDR}$ is well above unity, indicating that there is room for improvement.

\section{B. Roy equations}

Roy equations are fully equivalent to nonforward dispersion relations, plus some $t-s$ crossing symmetry, projected on the various partial waves. They can be written as

$$
\begin{aligned}
\operatorname{Re} f_{l}^{(I)}(s)= & C_{l}^{(I)} a_{0}^{(0)}+C_{l}^{(I)} a_{0}^{(2)} \\
& +\sum_{l^{\prime}, I^{\prime}} \text { P.P. } \int_{4 M_{\pi}^{2}}^{\infty} \mathrm{d} s^{\prime} K_{l, l^{\prime} ; I, I^{\prime}}\left(s^{\prime}, s\right) \operatorname{Im} f_{l^{\prime}}^{\left(I^{\prime}\right)}\left(s^{\prime}\right) .
\end{aligned}
$$

$C_{l}^{(I)}, C_{l}^{(I)}$ are known constants, and the kernels $K_{l, l^{\prime} ; I, I^{\prime}}$ are also known.

We also define the quantities

$$
\begin{aligned}
\Delta_{l}^{(I)}(s) \equiv & \operatorname{Re} f_{l}^{(I)}(s)-C_{l}^{(I)} a_{0}^{(0)}-C_{l}^{(I)} a_{0}^{(2)} \\
& -\sum_{l^{\prime}, I^{\prime}} \text { P.P. } \int_{4 M_{\pi}^{2}}^{\infty} \mathrm{d} s^{\prime} K_{l, l^{\prime} ; I, I^{\prime}}\left(s^{\prime}, s\right) \operatorname{Im} f_{l^{\prime}}^{\left(I^{\prime}\right)}\left(s^{\prime}\right) ;
\end{aligned}
$$

they would vanish if Roy equations were exactly fulfilled.

Roy equations are only valid up to $s=64 M_{\pi}^{2} \simeq 1 \mathrm{GeV}^{2}$ because, for larger values, the integrand receives new contributions from the double spectral functions, not contained in (4.5). Moreover, when the value of $t$ over which one integrates to project the partial waves is $|t| \gg \Lambda_{\mathrm{QCD}}^{2}$, for $s \simeq 2 \mathrm{GeV}^{2}$, the Regge expressions are not valid. This is a further limitation of the validity of the Roy equations to energies below $\sim 1 \mathrm{GeV}$. 

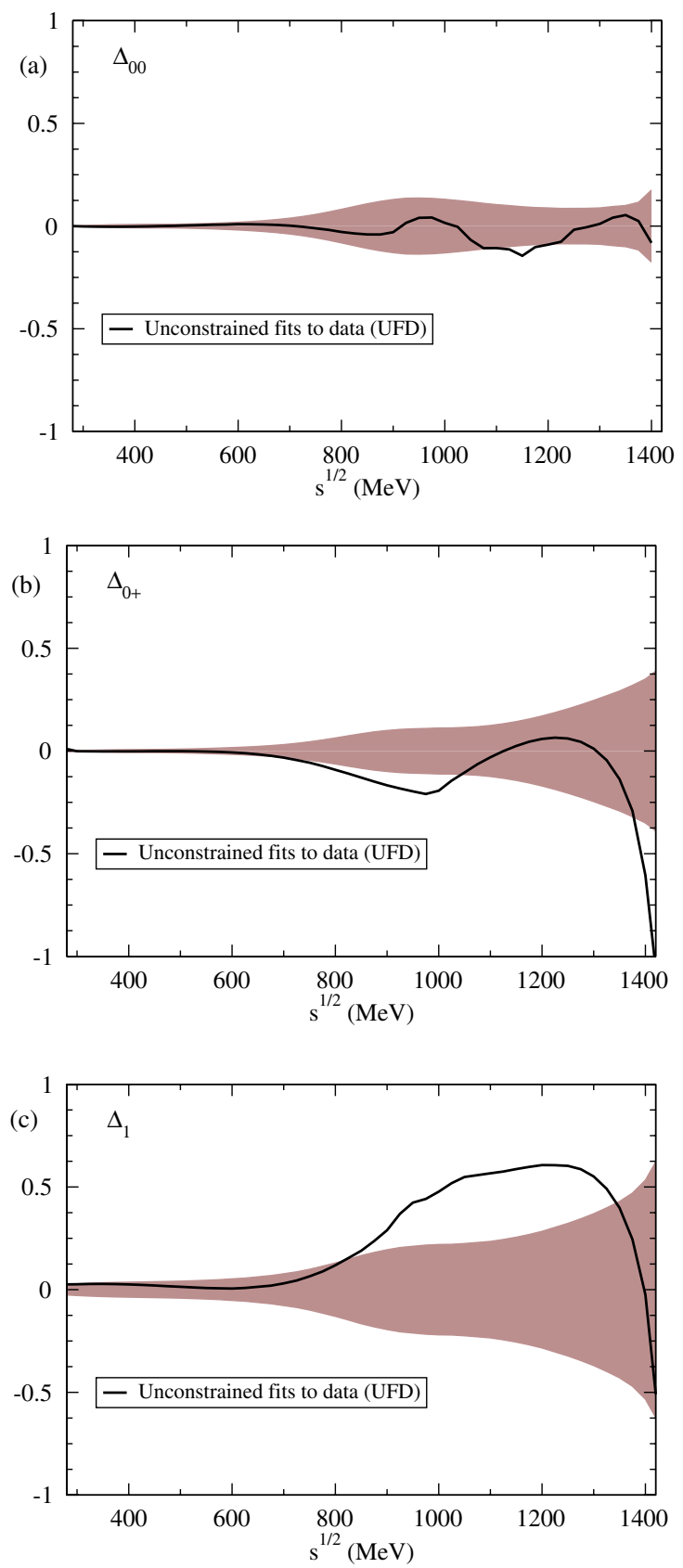

FIG. 5 (color online). Fulfillment of dispersion relations using the UFD amplitudes. From top to bottom, the continuous line stands for the differences defined in Eqs. (4.2a)-(4.2c), respectively. The dark band covers the uncertainties.

We here calculate up to $\bar{K} K$ threshold, and only test the waves S0, S2, P. ${ }^{6}$ We define the equivalent of the average discrepancies $\bar{d}^{2}$ we used for the forward dispersion relations,

\footnotetext{
${ }^{6}$ A preliminary review of these results was presented at the 4th International Conference on Quarks and Nuclear Physics, Madrid, June 2006 [12].
}

$$
\bar{d}_{l, I}^{2} \equiv \frac{1}{\text { number of points }} \sum_{n}\left(\frac{\Delta_{l}^{(I)}\left(s_{n}\right)}{\delta \Delta_{l}^{(I)}\left(s_{n}\right)}\right)^{2},
$$

and find the results shown in Fig. 6, where we plot what our parametrizations give for $\operatorname{Re} f_{l}^{(I)}$ (denoted by "in") and what follows from the integrals in the right-hand side of (4.5a), denoted by "out."
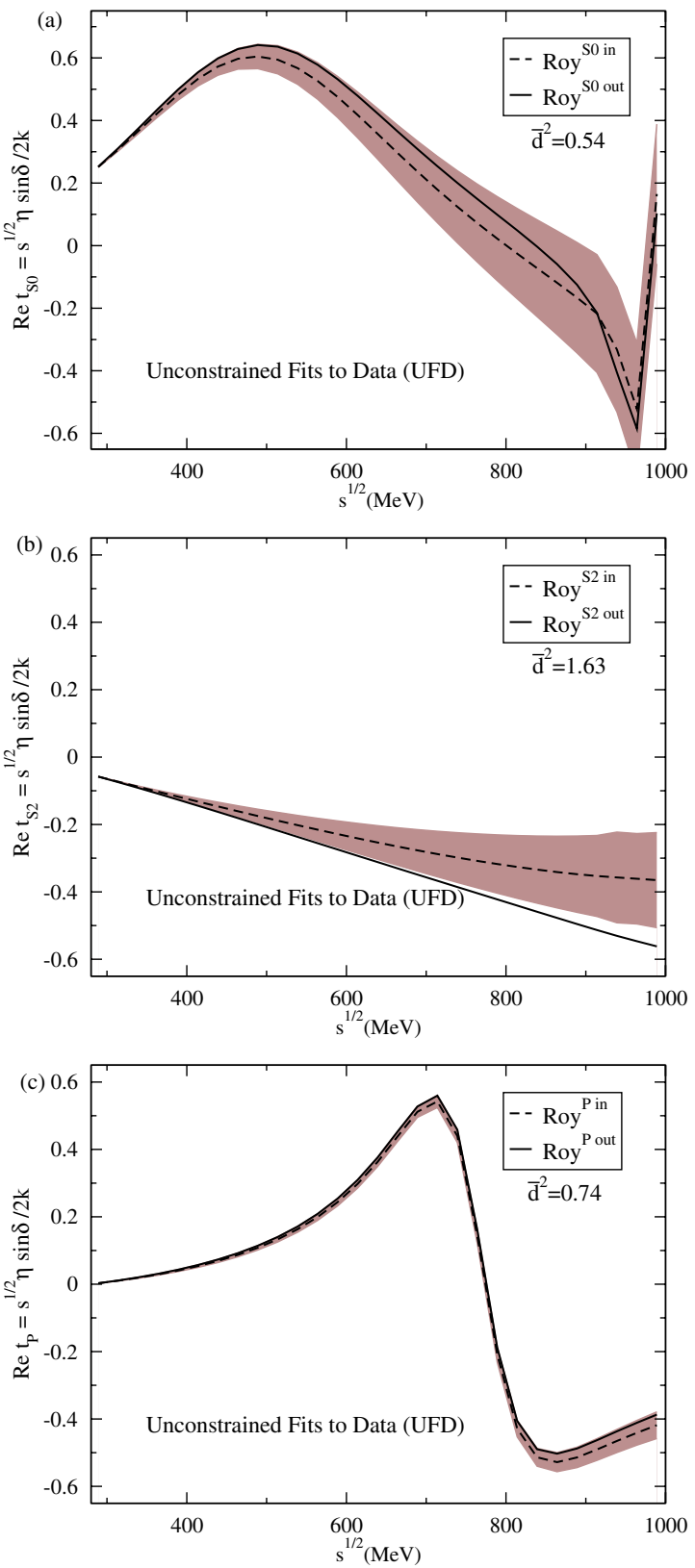

FIG. 6 (color online). Results for Roy equations using the UFD amplitudes. The continuous lines stand for the result from the dispersive integral and the dashed lines for the real part. The dark band covers the uncertainty in the difference between both. From top to bottom: (a) S0 wave, (b) S2 wave, (c) $\mathrm{P}$ wave. 
Numerically, we have the results

$$
\bar{d}_{S 0}^{2}=0.54, \quad \bar{d}_{S 2}^{2}=1.63, \quad \bar{d}_{P}^{2}=0.74:
$$

a reasonable fulfillment, but it is clear that this can be improved, particularly for the S2 wave for which the discrepancy is larger than unity.

\section{IMPROVEMENT OF THE PARAMETRIZATIONS OF THE PARTIAL WAVES: FITS TO DATA CONSTRAINED REQUIRING FULFILLMENT OF DISPERSION RELATIONS}

\section{A. Two sum rules}

Apart from forward dispersion relations and Roy equations, we will also require fulfillment, within errors, of two sum rules that relate high energy (Regge) parameters for $t \neq 0$ to low energy $\mathrm{P}$ and $\mathrm{D}$ waves.

The first sum rule is (Ref. [1])

$$
\begin{aligned}
I \equiv & \int_{4 M_{\pi}^{2}}^{\infty} \mathrm{d} s \frac{\operatorname{Im} F^{\left(I_{t}=1\right)}\left(s, 4 M_{\pi}^{2}\right)-\operatorname{Im} F^{\left(I_{t}=1\right)}(s, 0)}{s^{2}} \\
& -\int_{4 M_{\pi}^{2}}^{\infty} \mathrm{d} s \frac{8 M_{\pi}^{2}\left[s-2 M_{\pi}^{2}\right]}{s^{2}\left(s-4 M_{\pi}^{2}\right)^{2}} \operatorname{Im} F^{\left(I_{s}=1\right)}(s, 0)=0 .
\end{aligned}
$$

The contributions of the $S$ waves cancel in (5.1), so only the P, D, F, and G waves contribute. At high energy the integrals are dominated by rho exchange.

The second sum rule we consider is that given in Eqs. (B.6), (B.7) of Ref. [13]. It reads

$$
\begin{aligned}
J \equiv & \int_{4 M_{\pi}^{2}}^{\infty} \mathrm{d} s\left\{\frac{4 \operatorname{Im} F^{\prime(0)}(s, 0)-10 \operatorname{Im} F^{\prime(2)}(s, 0)}{s^{2}\left(s-4 M_{\pi}^{2}\right)^{2}}\right. \\
& \left.-6\left(3 s-4 m_{\pi}^{2}\right) \frac{\operatorname{Im} F^{\prime(1)}(s, 0)-\operatorname{Im} F^{(1)}(s, 0)}{s^{2}\left(s-4 M_{\pi}^{2}\right)^{3}}\right\}=0 .
\end{aligned}
$$

Here $F^{(I)}(s, t) \equiv \partial F^{(I)}(s, t) / \partial \cos \theta$. At high energy, the integral is dominated by isospin zero Regge trajectories. We also define a discrepancy for these sum rules:

$$
\bar{d}_{I}^{2}=\left(\frac{I}{\delta I}\right)^{2}, \quad \bar{d}_{J}^{2}=\left(\frac{J}{\delta J}\right)^{2} .
$$

These two sum rules are reasonably satisfied, if using the partial wave parameters obtained from data (Sec. II), and the Regge parameters determined from factorization and fits to data (Sec. III).

\section{B. Minimization procedure}

Because forward dispersion relations and Roy equations are satisfied almost within the fluctuations induced by the experimental errors, it makes sense to repeat the fits to experiment requiring verification within errors of forward dispersion relations and Roy equations, to which we add the sum rules (5.1) and (5.2) to control Regge parameters away from the forward direction. We do this by minimizing the quantity $\chi^{2}$ defined, with self-evident notation, by

$$
\begin{aligned}
\chi^{2} \equiv & \left\{\bar{d}_{00}^{2}+\bar{d}_{0+}^{2}+\bar{d}_{I_{t}=1}^{2}+\bar{d}_{S 0}^{2}+\bar{d}_{S 2}^{2}+\bar{d}_{P}^{2}\right\} W+\bar{d}_{I}^{2}+\bar{d}_{J}^{2} \\
& +\sum_{i}\left(\frac{p_{i}-p_{i}^{\exp }}{\delta p_{i}}\right)^{2} .
\end{aligned}
$$

Here, $p_{i}^{\exp }$ are the parameters that we have found in the unconstrained fits to experimental data, and $\delta p_{i}$ are their errors. Thus the sum over $p_{i}$ runs over $B_{n} \mathrm{~s}$, zeros $\left(z_{0}, z_{2}, \Delta\right)$, inelasticity parameters $\epsilon_{n}, r$, etc., and over the $\mathrm{K}$-matrix parameters for the $\mathrm{S} 0$ wave. The presence of the $\operatorname{sum} \sum_{i}\left[\left(p_{i}-p_{i}^{\exp }\right) / \delta p_{i}\right]^{2}$, of course, ensures fit to experimental data.

The quantity $W$ in (5.4) is a weight, which can be estimated in two different manners. First, it will serve to give each of the dispersion relations (FDR or Roy) a weight appropriate to the information that they carry. For example, for the FDR for $\pi^{0} \pi^{0}$ scattering, the quantity $\operatorname{Re} F_{00}(s)-$ $F_{00}\left(4 M_{\pi}^{2}\right)$ can be fixed giving the slope at $s=4 M_{\pi}^{2}$, the value of $\operatorname{Re} F_{00}(s)-F_{00}\left(4 M_{\pi}^{2}\right)$ and its derivative at each of the points $s$ where its changes direction; and the same at the end point, $s=1.42 \mathrm{GeV}^{2}$ (see Fig. 4(a)): altogether, 13 values. Any reasonably smooth function that fits these 13 values is sure to follow $\operatorname{Re} F_{00}(s)-F_{00}\left(4 M_{\pi}^{2}\right)$ in all the range: putting extra weight would be imposing redundant constraints. For other dispersion relations the number is a bit smaller; in general, a number $6 \leqq W \leqq 13$ is obtained. An alternate method to find $W$ is to increase it so that all dispersion relations are satisfied within errors, that is to say, all the corresponding $\bar{d}^{2}$ are less than or equal to unity. This occurs for $W \sim 9$. In our calculation we have taken $W=9$, although we have verified that results practically indistinguishable are obtained for $7 \leq W \leq 12$.

\section{Results for the constrained fits (CFD)}

The results of the fits to data, constrained by requiring fulfillment of the FDR and Roy equations plus the sum rules (5.1) and (5.2), that we call set CFD, are summarized in Appendix B. This CFD set is obtained by minimizing $\chi^{2}$ as defined in Eq. (5.4). In general, the parameters hardly change with respect to what we had from fits to data, Appendix A; but there are a few waves for which there are noteworthy alterations. First of all, we have the S0 and $\mathrm{S} 2$ waves. Because now we are requiring verification of the FDR below threshold, we can leave the location of the Adler zeros $z_{0}, z_{2}$ free. This produces some changes in the parameters $B_{n}$, since there is a strong correlation between them and the location of the Adler zeros. However, the phase shifts themselves are practically identical to what we had in fits to data, Appendix A. Then we have the D2 wave, the only one that changes appreciably. It moves by a bit more than 1 standard deviation. The S2 wave also moves appreciably after constraining its fit; we discuss the two 
later on (Secs. VD and VE). All other waves change so little that the difference between sets UDF and CFD is almost inappreciable.

For the present CFD set, FDRs and Roy equations are, of course, better satisfied than before. For the FDR we find

$$
\begin{array}{cccc} 
& & s^{1 / 2} \leq 932 \mathrm{MeV} & s^{1 / 2} \leq 1420 \mathrm{MeV} \\
\pi^{0} \pi^{0} & \text { FDR } & \bar{d}^{2}=0.13 & \bar{d}^{2}=0.31 \\
\pi^{0} \pi^{+} & \text {FDR } & \bar{d}^{2}=0.83 & \bar{d}^{2}=0.85 \\
I_{t}=1 & \text { FDR } & \bar{d}^{2}=0.13 & \bar{d}^{2}=0.70,
\end{array}
$$

and, for the Roy equations,

$$
\bar{d}_{S 0}^{2}=0.23, \quad \bar{d}_{S 2}^{2}=0.25, \quad \bar{d}_{P}^{2}=2 \times 10^{-3} .
$$

Furthermore, for the sum rules (5.1) and (5.2) we have $\bar{d}_{I}^{2}=0.02$ and $\bar{d}_{J}^{2}=0.55$. The verification of FDR and Roy equations are shown graphically in Figs. 7-9.

The overall average $\bar{d}^{2}$ is now substantially smaller than unity; particularly for the Roy equations. However, for the FDR for $\pi^{0} \pi^{+}$scattering is near unity. This is probably due to the D2 wave, which is likely still a bit away from its correct location, and to the $\mathrm{P}$ wave at energies above $1380 \mathrm{MeV}$, where our parametrization fails to take account of the $\rho(1450)$ resonance. This indicates that one is at the limit of accuracy for experimentally-based parametrizations of scattering amplitudes.

Besides sum rules and dispersion relations, another independent test of our amplitudes is the Adler sum rule that relates the pion decay constant to pion-pion scattering amplitudes with one pion off its mass shell. This has been recently evaluated[14] with our scattering UFD amplitudes, and a very satisfactory fulfillment of the sum rule is found; the discrepancy $\Delta_{\pi}$ that measures the accuracy with which the sum rule is fulfilled (and which should vanish if it was satisfied exactly) is found to be $\Delta_{\pi}=$ $0.021 \pm 0.053$.

\section{Results: Comparison of UFD and CFD sets}

We here present the comparison of our sets UFD and CFD, i.e., what we have by directly fitting data, and what is obtained constraining the fits by imposing also FDRs and Roy equations. ${ }^{7}$ Note that in the CFD set we have here only altered the central values; we leave the errors that follow from fits to data, i.e., we assume errors as in set UFD. Note also that, in the following formulas, the parameters are as defined in Appendices A and B.

\footnotetext{
${ }^{7}$ For the S0 wave, we give here only the values obtained neglecting isospin breaking; the values of the parameters obtained taking isospin breaking into account may be found in Appendix B.
}
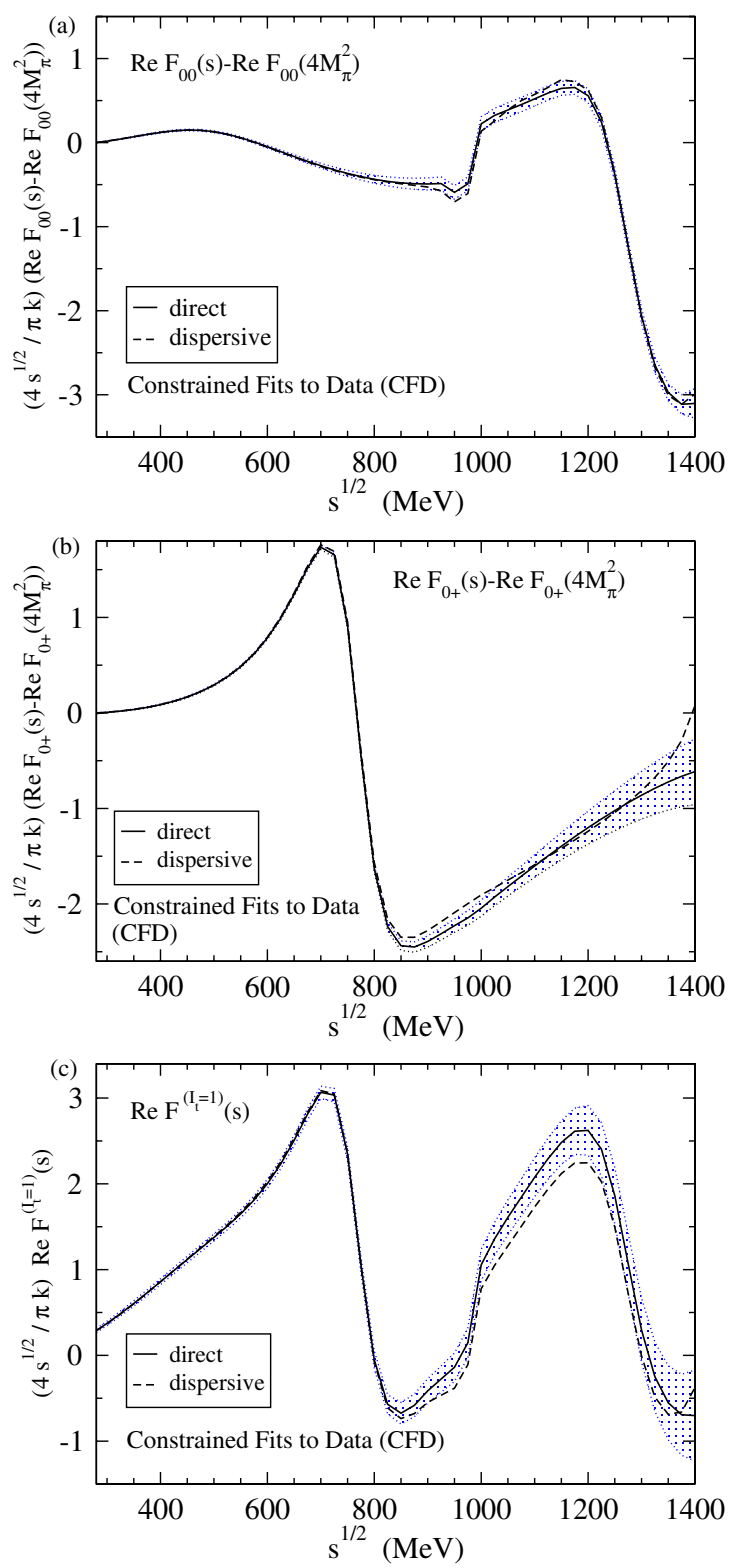

FIG. 7 (color online). Results for forward dispersion relations. Continuous lines: real part, evaluated directly with the CFD parametrizations. Dashed lines: the result of the dispersive integrals. The gray bands cover the uncertainties in the difference between both. (a) The $\pi^{0} \pi^{0}$ FDR, (b) the $\pi^{0} \pi^{+}$FDR, (c) the FDR for $I_{t}=1$ scattering.

SO wave. We have, for $\mathrm{S} 0$ below $932 \mathrm{MeV}$,

$$
\begin{array}{ccc} 
& \mathrm{UFD}, z_{0} \equiv M_{\pi} & \mathrm{CFD}, z_{0} \text { free } \\
B_{0} & 4.3 \pm 0.3 & 4.41 \pm 0.3 \\
B_{1} & -26.7 \pm 0.6 & -26.25 \pm 0.6 \\
B_{2} & -14.1 \pm 1.4 & -15.8 \pm 1.4 \\
z_{0} & M_{\pi} & 166.1 \pm 4.2 \mathrm{MeV} .
\end{array}
$$

Above $932 \mathrm{MeV}$, 
PION-PION SCATTERING AMPLITUDE. III. ...
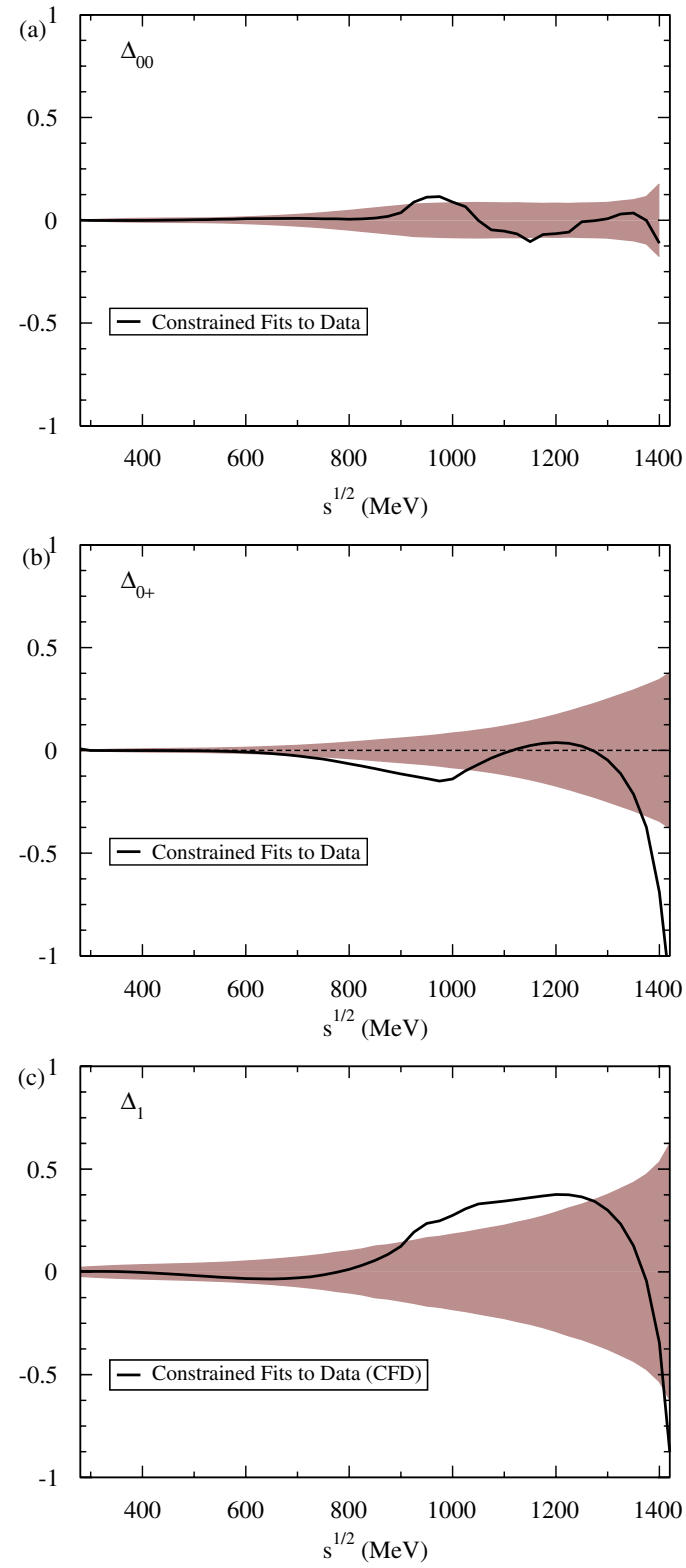

FIG. 8 (color online). Fulfillment of dispersion relations using the CFD amplitudes. From top to bottom, the continuous line stands for the differences defined in Eqs. (4.2a)-(4.2c), respectively. The dark band covers the uncertainties.

UFD

CFD

$\begin{array}{ccc}\alpha_{1} & 0.843 \pm 0.017 & 0.843 \pm 0.017 \\ \alpha_{2} & 0.20 \pm 0.06 & 0.20 \pm 0.06 \\ \beta_{1} & 1.02 \pm 0.02 & 1.02 \pm 0.02 \\ \beta_{2} & 1.33 \pm 0.013 & 1.33 \pm 0.013 \\ \gamma_{11} & 3.10 \pm 0.11 & 3.10 \pm 0.11 \\ \gamma_{12} & 1.82 \pm 0.05 & 1.81 \pm 0.05 \\ \gamma_{22} & -7.00 \pm 0.04 & -7.00 \pm 0.04 \\ M_{1} & 888 \pm 4 \mathrm{MeV} & 888 \pm 4 \\ M_{2} & 1327 \pm 4 \mathrm{MeV} & 1327 \pm 4 .\end{array}$

PHYSICAL REVIEW D 77, 054015 (2008)
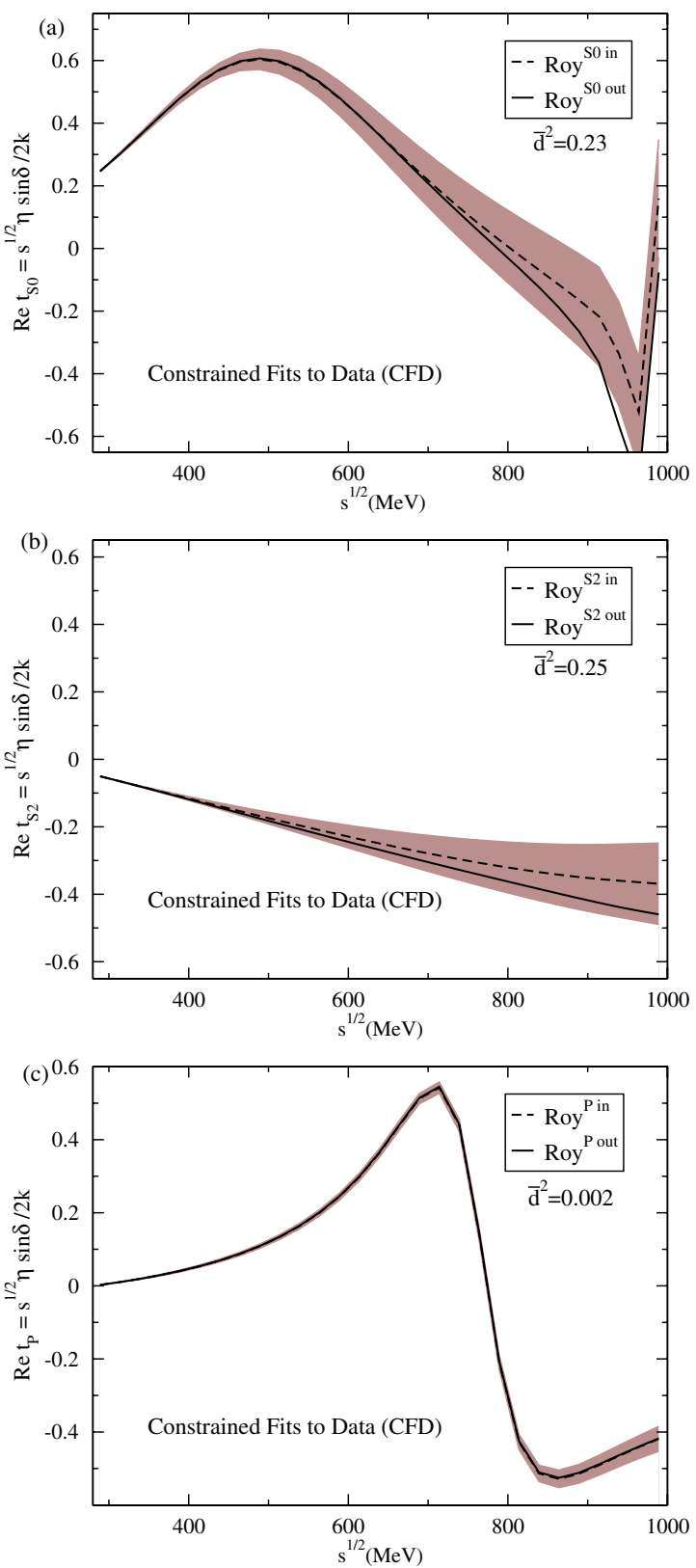

FIG. 9 (color online). Results for Roy equations using the CFD amplitudes. The continuous lines stand for the result from the dispersive integral and the dashed lines for the real part. The dark band covers the uncertainty in the difference between both. From top to bottom: (a) S0 wave, (b) S2 wave, (c) P wave.

S2 wave. We now find

$$
\begin{array}{ccc} 
& \mathrm{UFD}, z_{0} \equiv M_{\pi} & \mathrm{CFD}, z_{2} \text { free } \\
B_{0} & -80.4 \pm 2.8 & -80.2 \pm 2.8 \\
B_{1} & -73.6 \pm 10.5 & -69.4 \pm 10.5 \\
B_{h 2} & 109 \pm 38 & 120 \pm 38 \\
z_{2} & M_{\pi} & 145.0 \pm 3.6 \mathrm{MeV} .
\end{array}
$$

For the inelasticity, 


$$
\begin{array}{ccc} 
& \text { UFD } & \text { CFD } \\
\epsilon & 0.17 \pm 0.12 & 0.18 \pm 0.12 .
\end{array}
$$

$P$ wave. In this case we have kept the value of the $\rho$ resonance mass fixed when imposing dispersion relations; thus, for both sets UFD and CFD, $M_{\rho}=773.6 \pm$ $0.9 \mathrm{MeV}$. For the remaining parameters below $992 \mathrm{MeV}$ we find

$$
\begin{array}{ccc} 
& \text { UFD } & \text { CFD } \\
B_{0} & 1.055 \pm 0.011 & 1.052 \pm 0.011 \\
B_{1} & 0.15 \pm 0.05 & 0.17 \pm 0.05
\end{array}
$$

Above $992 \mathrm{MeV}$,

$$
\begin{array}{ccc} 
& \text { UFD } & \text { CFD } \\
\lambda_{1} & 1.57 \pm 0.18 & 1.50 \pm 0.18 \\
\lambda_{2} & -1.96 \pm 0.49 & -1.97 \pm 0.49 \\
\epsilon_{1} & 0.10 \pm 0.06 & 0.09 \pm 0.06 \\
\epsilon_{2} & 0.11 \pm 0.11 & 0.12 \pm 0.11
\end{array}
$$

D0 wave. We here keep the mass of the resonance fixed at $M_{f_{2}}=1275.4 \mathrm{MeV}$ for both sets UFD and CFD. We have, below $992 \mathrm{MeV}$,

$$
\begin{array}{ccc} 
& \text { UFD } & \text { CFD } \\
B_{0} & 12.47 \pm 0.12 & 12.48 \pm 0.12 \\
B_{1} & 10.12 \pm 0.16 & 10.12 \pm 0.16
\end{array}
$$

Above $992 \mathrm{MeV}$,

$$
\begin{array}{ccc} 
& \text { UFD } & \text { CFD } \\
B_{h 1} & 43.7 \pm 1.8 & 43.5 \pm 1.8 \\
\epsilon_{1} & 0.284 \pm 0.030 & 0.283 \pm 0.030 \\
r & 2.54 \pm 0.31 & 2.53 \pm 0.31 .
\end{array}
$$

D2 wave. This is the only wave that changes substantially; see Fig. 10. We find now

UFD

$$
\begin{array}{ccc}
B_{0} & (2.4 \pm 0.5) \times 10^{3} & (3.1 \pm 0.5) \times 10^{3} \\
B_{1} & (7.8 \pm 1.0) \times 10^{3} & (7.9 \pm 1.0) \times 10^{3} \\
B_{2} & (23.7 \pm 4.2) \times 10^{3} & (24.7 \pm 4.2) \times 10^{3} \\
\Delta & 196 \pm 25 \mathrm{MeV} & 205 \pm 25 \mathrm{MeV}
\end{array}
$$

For the inelasticity parameter,

$$
\begin{array}{ccc} 
& \text { UFD } & \text { CFD } \\
\epsilon & 0.2 \pm 0.2 & 0.15 \pm 0.2 .
\end{array}
$$

$F$ wave. This wave is unchanged within our precision:

$$
\begin{array}{ccc} 
& \text { UFD } & \text { CFD } \\
B_{0} & (1.09 \pm 0.03) \times 10^{5} & (1.09 \pm 0.03) \times 10^{5} \\
B_{1} & (1.41 \pm 0.04) \times 10^{5} & (1.41 \pm 0.04) \times 10^{5}
\end{array}
$$

Regge parameters. We only give the values of the Regge parameters that we have allowed to vary. The parameters correspond to the formulas in Sec. III.

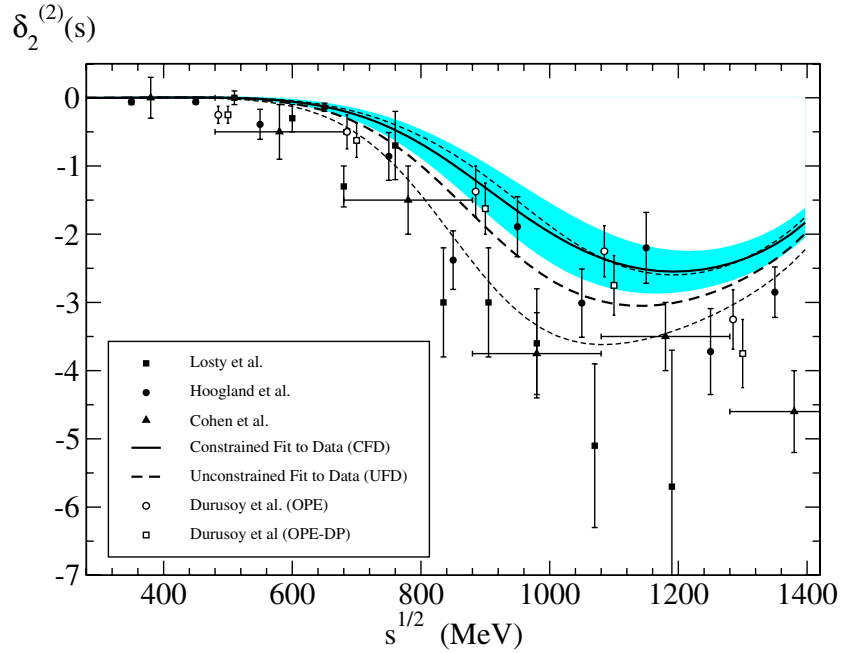

FIG. 10 (color online). The $I=2$, D wave phase shift. Dashed line: fit to data with Eq. (2.7d). Continuous line and shaded area: after improving with dispersion relations. Also shown are data points from Ref. [6].

UFD CFD

$$
\begin{array}{ccc}
c_{P} & (0.0 \pm 1.0) \mathrm{GeV}^{-2} & (0.53 \pm 1.0) \mathrm{GeV}^{-2} \\
c_{P^{\prime}} & -0.4 \pm 0.4 \mathrm{GeV}^{-2} & -0.38 \pm 0.4 \mathrm{GeV}^{-2} \\
\beta_{P^{\prime}} & 0.83 \pm 0.05 & 0.83 \pm 0.05 \\
\alpha_{P^{\prime}}(0) & 0.54 \pm 0.02 & 0.54 \pm 0.02 \\
\beta_{\rho} & 1.22 \pm 0.14 & 1.30 \pm 0.14 \\
\alpha_{\rho}(0) & 0.46 \pm 0.02 & 0.46 \pm 0.02 \\
\beta_{2} & 0.20 \pm 0.2 & 0.22 \pm 0.2 .
\end{array}
$$

The only parameters that change appreciably (but both by only $\sim 0.5 \sigma)$ are $c_{P}$, which was to be expected, and $\beta_{\rho}$.

\section{E. Comments}

From Eqs. (5.7) through (5.13) we see that the changes in most waves induced by the constraints given by FDR and Roy equations are very small; in many cases, minute or even nonexistent within the accuracy of our formulas. There are a few exceptions. First of all, the $B_{n}$ s for the S0 and S2 waves change because now the location of the Adler zeros is left free (although the phase shifts themselves move very little). Second, the $B_{0}$ and $B_{1}$ parameters of the $\mathrm{P}$ wave vary by 0.3 and $0.4 \sigma$ respectively, and, at high energy, the parameter $\lambda_{1}$ changes by $0.3 \sigma$. And third, the only wave that suffers changes by more than one sigma is the $\mathrm{D} 2$ wave, as was to be expected; the parameter, $B_{0}$, moves by $1.5 \sigma$. All the other parameters of the $\mathrm{S} 0, \mathrm{~S} 2$, and $\mathrm{P}$ waves, as well as all the parameters of D0, F waves, change below the limit of relevance.

Altogether, the stability of the fit against imposing FDR and Roy equations is remarkable, showing its robustness. The stability is not obtained at the cost of large errors; quite 


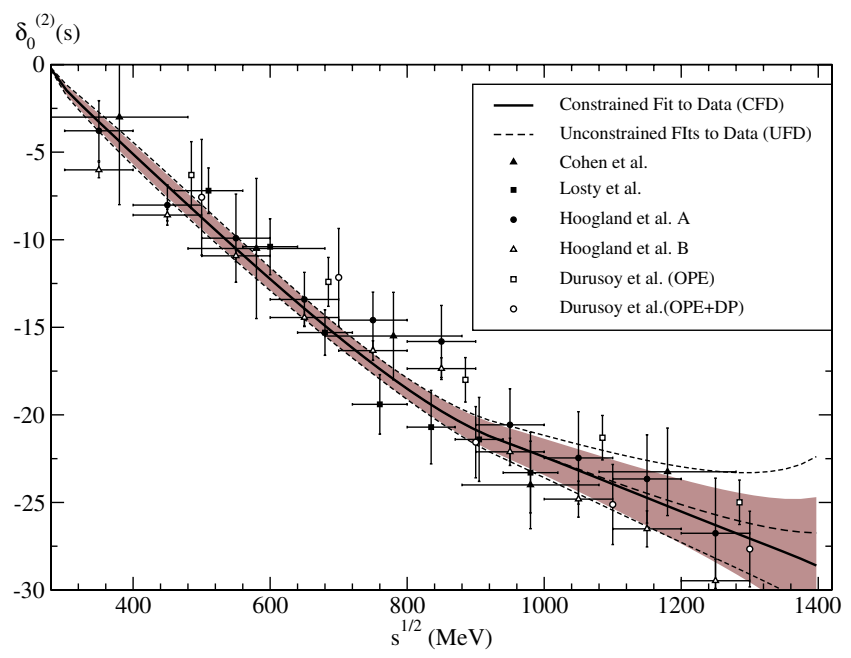

FIG. 11 (color online). The $I=2, S$-wave phase shift. Dashed line: fit to data with Eqs. (2.2) and (2.3). Continuous line and shaded area: after improving with dispersion relations. Also shown are data points from Ref. [6].

the contrary. Except for the S2 wave at intermediate energies, that we show in Fig. 11, and for the D2 wave, where the errors are larger, the errors in the other waves are as small (for the P, F waves) or much smaller (by a factor $\sim 3$ ) than what we had in previous fits, in Ref. [1,2], even after improving with FDR.

Finally, a few words may be said on the Regge parameters. The parameters for exchange of isospin zero are almost unchanged when requiring fit to FDR, Roy equations, and sum rules. This was to be expected; they are very well determined from $\pi N$ and $N N$ amplitudes using factorization. For exchange of isospin unity, only the parameter $\beta_{\rho}$ changes appreciably, and this by $\sim 0.5 \sigma$. This shows the high degree of compatibility between our amplitudes above and below $1420 \mathrm{MeV}$.

\section{LOW ENERGY PARAMETERS AND OTHER OBSERVABLES}

\section{A. General}

We present in Table I the low energy parameters (scattering lengths and effective range parameters, in units of the charged pion mass) that follow from our calculations. Besides scattering lengths and effective range parameters, defined as

$$
\begin{gathered}
\frac{s^{1 / 2}}{2 M_{\pi} k^{2 l+1}} \operatorname{Re} \hat{f}_{l}^{(I)}(s) \simeq_{k \rightarrow 0} a_{l}^{(I)}+b_{l}^{(I)} k^{2}+\cdots, \\
\hat{f}_{l}^{(I)}=\sin \delta_{l}^{(I)} \mathrm{e}^{\mathrm{i} \delta_{l}^{(I)}}, \quad k=\sqrt{s / 4-M_{\pi}^{2}},
\end{gathered}
$$

where $\hat{f}_{l}^{(I)}$ is the partial wave of definite isospin $I$ and angular momentum $l$ (in the elastic region) and $\delta_{l}^{(I)}$ stands for its corresponding phase shift, we give the quantities $a_{0}^{(0)}-a_{0}^{(2)}$ and $\delta_{0}^{(0)}\left(m_{K}^{2}\right)-\delta_{0}^{(2)}\left(m_{K}^{2}\right)$. These quantities are relevant for pionic atom decays and kaon decays. We also give the combination $2 a_{0}^{(0)}-5 a_{0}^{(2)}$ that appears in the Olsson sum rule,

$$
2 a_{0}^{(0)}-5 a_{0}^{(2)}=3 M_{\pi} \int_{4 M_{\pi}^{2}}^{\infty} \mathrm{d} s \frac{\operatorname{Im} F^{\left(I_{t}=1\right)}(s, 0)}{s\left(s-4 M_{\pi}^{2}\right)}
$$

which corresponds to the $I_{t}=1$ amplitude forward dispersion relation evaluated at threshold. This sum rule is very useful in determining a precise value for $a_{0}^{(2)}$.

Besides the Olsson sum rule, the results from direct fits may be improved with the help of the Froissart-Gribov representation (whose explicit form may be found in Ref. [1]), and the following sum rules: the sum rule [Eq. (6.8)] in Ref. [1], which we repeat here in Eq. (6.2) for ease of reference,

$$
\begin{aligned}
b_{1}= & \frac{2}{3 M_{\pi}} \int_{4 M_{\pi}^{2}}^{\infty} \mathrm{d} s\left\{\frac{1}{3}\left[\frac{1}{\left(s-4 M_{\pi}^{2}\right)^{3}}-\frac{1}{s^{3}}\right] \operatorname{Im} F^{\left(I_{t}=0\right)}(s, 0)\right. \\
& +\frac{1}{2}\left[\frac{1}{\left(s-4 M_{\pi}^{2}\right)^{3}}+\frac{1}{s^{3}}\right] \operatorname{Im} F^{\left(I_{t}=1\right)}(s, 0) \\
& \left.-\frac{5}{6}\left[\frac{1}{\left(s-4 M_{\pi}^{2}\right)^{3}}-\frac{1}{s^{3}}\right] \operatorname{Im} F^{\left(I_{t}=2\right)}(s, 0)\right\}
\end{aligned}
$$

and two sum rules involving the effective range parameters for the S0, S2 waves. These are obtained evaluating the limit as $s \rightarrow 4 M_{\pi}^{2}$ of the ratio

$$
\frac{F(s, 0)-F\left(4 M_{\pi}^{2}, 0\right)}{s\left(s-4 M_{\pi}^{2}\right)}
$$

in the forward dispersion relations for $\pi^{0} \pi^{0}$ and $\pi^{0} \pi^{+}$ scattering. We find

$$
\begin{aligned}
& b_{0}^{(0)}+2 b_{0}^{(2)}=6 M_{\pi} \lim _{\substack{s \rightarrow 4 M_{\pi}^{2} \\
s>4 M_{\pi}^{2}}} \text { P.P. } \int_{4 M_{\pi}^{2}}^{\infty} \mathrm{d} s^{\prime} \frac{\left(2 s^{\prime}-4 M_{\pi}^{2}\right) \operatorname{Im} F_{00}\left(s^{\prime}\right)}{s^{\prime}\left(s^{\prime}+s-4 M_{\pi}^{2}\right)\left(s^{\prime}-4 M_{\pi}^{2}\right)\left(s^{\prime}-s\right)}, \\
& 3 a_{1}^{(1)}+b_{0}^{(2)}=4 M_{\pi} \lim _{\substack{s \rightarrow 4 M_{\pi}^{2} \\
s>4 M_{\pi}^{2}}} \text { P.P. } \int_{4 M_{\pi}^{2}}^{\infty} \mathrm{d} s^{\prime} \frac{\left(2 s^{\prime}-4 M_{\pi}^{2}\right) \operatorname{Im} F_{0+}\left(s^{\prime}\right)}{s^{\prime}\left(s^{\prime}+s-4 M_{\pi}^{2}\right)\left(s^{\prime}-4 M_{\pi}^{2}\right)\left(s^{\prime}-s\right)} .
\end{aligned}
$$


TABLE I. Units of $M_{\pi}$. For the best values for $a_{1}, b_{1}, b_{0}^{(0)}$, and $b_{0}^{(2)}$ we have averaged what comes from constrained fits (CFD), with what one finds from the sum rules (since they are practically independent). The best values for the other parameters are as follows: from the CFD, for $a_{0}^{(0)}$. For D0, D2, and F waves, the best values are those coming from the Froissart-Gribov representation; because our fits to data impose these values, it would not make sense to average them. However, we have averaged the results for $b_{2}^{(2)}$ and $b_{3}$ since we did not impose their values when fitting the data.

\begin{tabular}{lcccc}
\hline \hline & Unconstrained fits (UFD) & Constrained (CFD) & Sum rules, with CFD & Best values \\
\hline$a_{0}^{(0)}$ & $0.231 \pm 0.009$ & $0.223 \pm 0.010$ & $0.223 \pm 0.009$ \\
$a_{0}^{(2)}$ & $-0.052 \pm 0.010$ & $-0.0451 \pm 0.0088$ & $-0.0444 \pm 0.0045^{\mathrm{e}}$ \\
$a_{0}^{(0)}-a_{0}^{(2)}$ & $0.282 \pm 0.014$ & $0.268 \pm 0.014$ & $0.267 \pm 0.009^{\mathrm{f}}$ \\
$2 a_{0}^{(0)}-5 a_{0}^{(2)}$ & $0.720 \pm 0.055$ & $0.672 \pm 0.048$ & $0.667 \pm 0.018^{\mathrm{a}}$ & $0.668 \pm 0.017$ \\
$\delta_{0}^{(0)}\left(m_{K}^{2}\right)-\delta_{0}^{(2)}\left(m_{K}^{2}\right)$ & $51.7 \pm 1.2^{\circ}$ & $50.9 \pm 1.2^{\circ}$ & & $50.9 \pm 1.2^{\circ}$ \\
$b_{0}^{(0)}$ & $0.288 \pm 0.009$ & $0.291 \pm 0.009$ & $0.289 \pm 0.008^{\mathrm{d}}$ & $0.290 \pm 0.006$ \\
$b_{0}^{(2)}$ & $-0.085 \pm 0.010$ & $-0.084 \pm 0.010$ & $-0.081 \pm 0.0035^{\mathrm{d}}$ & $-0.081 \pm 0.003$ \\
$a_{1}\left(\times 10^{3}\right)$ & $37.3 \pm 1.2$ & $38.0 \pm 1.2$ & $38.2 \pm 1.3^{\mathrm{b}}$ & $38.1 \pm 0.9$ \\
$b_{1}\left(\times 10^{3}\right)$ & $5.18 \pm 0.23$ & $5.09 \pm 0.25$ & $5.42 \pm 0.91^{\mathrm{b}}, 5.13 \pm 0.19^{\mathrm{c}}$ & $5.12 \pm 0.15$ \\
$a_{2}^{(0)}\left(\times 10^{4}\right)$ & $18.7 \pm 0.4$ & $18.7 \pm 0.4$ & $18.33 \pm 0.36^{\mathrm{b}}$ & $18.33 \pm 0.36$ \\
$a_{2}^{(2)}\left(\times 10^{4}\right)$ & $2.5 \pm 1.1$ & $2.4 \pm 0.9$ & $-36 \pm 0.25^{\mathrm{b}}$ & $2.46 \pm 0.25$ \\
$b_{2}^{(0)}\left(\times 10^{4}\right)$ & $-4.2 \pm 0.3$ & $-4.2 \pm 0.3$ & $-3.64 \pm 0.25^{\mathrm{b}}$ & $-3.82 \pm 0.25$ \\
$b_{2}^{(2)}\left(\times 10^{4}\right)$ & $-2.7 \pm 1.0$ & $-2.5 \pm 0.8$ & $6.05 \pm 0.29^{\mathrm{b}}$ & $-3.59 \pm 0.18$ \\
$a_{3}\left(\times 10^{5}\right)$ & $5.2 \pm 1.3$ & $-4.40 \pm 0.36^{\mathrm{b}}$ & $6.05 \pm 0.29$ \\
$b_{3}\left(\times 10^{5}\right)$ & $-4.7 \pm 2.6$ & $-4.8 \pm 2.7$ & $-4.41 \pm 0.36$ \\
\hline \hline
\end{tabular}

afrom the Olsson sum rule.

${ }^{b}$ from the Froissart-Gribov representation.

${ }^{c}$ from the sum rule in Eq. (6.2).

${ }^{\mathrm{d}}$ from the sum rules in Eq. (6.3).

ebtained composing the CFD values for $a_{0}^{(0)}, a_{0}^{(2)}$ with the best value for $2 a_{0}^{(0)}-5 a_{0}^{(2)}$ from the Olsson sum rule.

${ }^{\mathrm{f}}$ This number takes into account the "best values" for $a_{0}^{(0)}, a_{0}^{(2)}$ given above.

Note that the limit has to be taken for $s$ larger than $4 M_{\pi}^{2}$; for $s<4 M_{\pi}^{2}$, the derivative of $\operatorname{Re} f_{0}(s)$ diverges like (Constant)/ik. The principal part of the integrals is essential; the RHS in (6.3) is convergent at the lower limit of integration only because it is a principal part, and one thus has

$$
\text { P.P. } \int_{0}^{\infty} \mathrm{d} x \frac{1}{(x-y) \sqrt{x}}=0, \quad \text { for } y>0 .
$$

Taking the value $a_{1}=(38.2 \pm 1.3) \times 10^{-3} M_{\pi}^{-3}$ from the Froissart-Gribov representation, with CFD waves (cf. Table I) this gives

$$
\begin{aligned}
& b_{0}^{(0)}=0.289 \pm 0.008 M_{\pi}^{-3}, \\
& b_{0}^{(2)}=-0.081 \pm 0.0035 M_{\pi}^{-3} .
\end{aligned}
$$

\section{B. The scattering lengths $a_{0}^{(0)}, a_{0}^{(2)}$}

The results reported under the headings UFD, CFD in Table I are what is found by fitting experimental data on partial wave amplitudes. However, for the scattering lengths $a_{0}^{(0)}$ and $a_{0}^{(2)}$ one can improve the results using the Olsson sum rule. One takes the value of the combination $2 a_{0}^{(0)}-5 a_{0}^{(2)}$ from the integral in (6.1), which is practi- cally independent of that obtained fitting data, and thus sets the constraints

$$
\begin{aligned}
& a_{0}^{(0)}=0.223 \pm 0.010 \quad[\mathrm{CDF}] \\
& a_{0}^{(2)}=-0.0451 \pm 0.0088 \quad[\mathrm{CDF}],
\end{aligned}
$$

$2 a_{0}^{(0)}-5 a_{0}^{(2)}=0.667 \pm 0.018 \quad$ [Olsson sum rule]

(in units of $M_{\pi}$ ).

We can then fit the $a_{0}^{(0)}, a_{0}^{(2)}$ minimizing (6.5). The resulting errors are strongly correlated (as is well-known); the corresponding ellipse is shown in Fig. 12. We can uncorrelate the errors by using two new variables, $x, y$ with

$$
\begin{aligned}
& a_{0}^{(0)}=0.223+0.129 x+0.335 y \\
& a_{0}^{(2)}=-0.0444-0.335 x+0.129 y \\
& x=0 \pm 0.0087, \quad y=0 \pm 0.027 .
\end{aligned}
$$

This gives the central values, and errors, reported under the heading "Best values" in Table I,

$$
a_{0}^{(0)}=0.223 \pm 0.009, \quad a_{0}^{(2)}=-0.0444 \pm 0.0045 .
$$

This represents a reasonable improvement on the errors we 


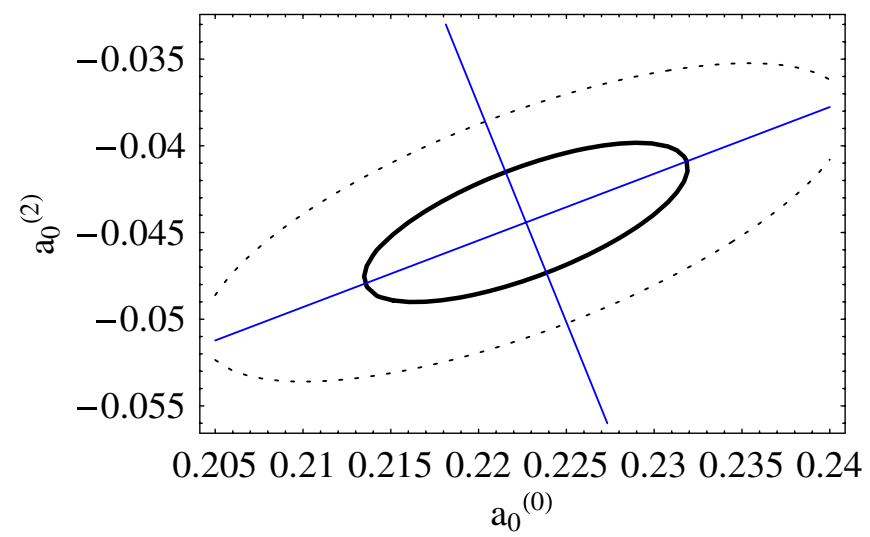

FIG. 12 (color online). The ellipses in the $a_{0}^{(0)}-a_{0}^{(2)}$ plane corresponding to 1-sigma (thick continuous line) and 2-sigma (broken line).

had before for $a_{0}^{(2)}$. We consider (6.7) to be our best result for these scattering lengths.

The S-waves scattering lengths can also be compared with other experimental information, not used in our fits, that give directly the combination $a_{0}^{(0)}-a_{0}^{(2)}$. Indeed, the value found in Table I for $a_{0}^{(0)}-a_{0}^{(2)}$ agrees very well with the following independent experimental determinations: from pionic atoms, [15] that give

$$
\left.a_{0}^{(0)}-a_{0}^{(2)}=0.280 \pm 0.013 \text { (St.) } \pm 0.008 \text { (Syst. }\right) M_{\pi}^{-1}
$$

and from $K_{3 \pi}$ decays that imply[16]

$$
\left.a_{0}^{(0)}-a_{0}^{(2)}=0.268 \pm 0.010(\text { St. }) \pm 0.013 \text { (Syst. }\right) M_{\pi}^{-1} .
$$

\section{CONCLUSIONS}

In the previous sections we have given a representation of the $\pi \pi$ scattering amplitudes obtained fitting experimental data below $1.42 \mathrm{GeV}$, supplemented by standard Regge formulas above this energy, what we have called the UFD set. We have shown that this UFD set satisfies very well forward dispersion relations and Roy equations, as well as crossing sum rules. Then, we have improved the central values of our fits requiring, besides fit to data, verification of FDR, Roy equations, and sum rules, getting what we have called CFD set. The central values in this CFD set lie well inside those of the UFD set, except for the D2 wave. FDR are now very well satisfied, while the verification of Roy equations is spectacular.

Altogether, we have confirmed the findings of Refs. [1,2] and (for the S0 wave) of Ref. [3]: but we have now errors much smaller than in Ref. [1]. At present, the low energy $\mathrm{P}$ wave is known at the limit of validity of our formalism (a limit given by isospin breaking effects, ${ }^{8}$ that our analysis neglects); while the S0 and D0 wave are near

\footnotetext{
${ }^{8}$ Some isospin breaking effects will be discussed below.
}
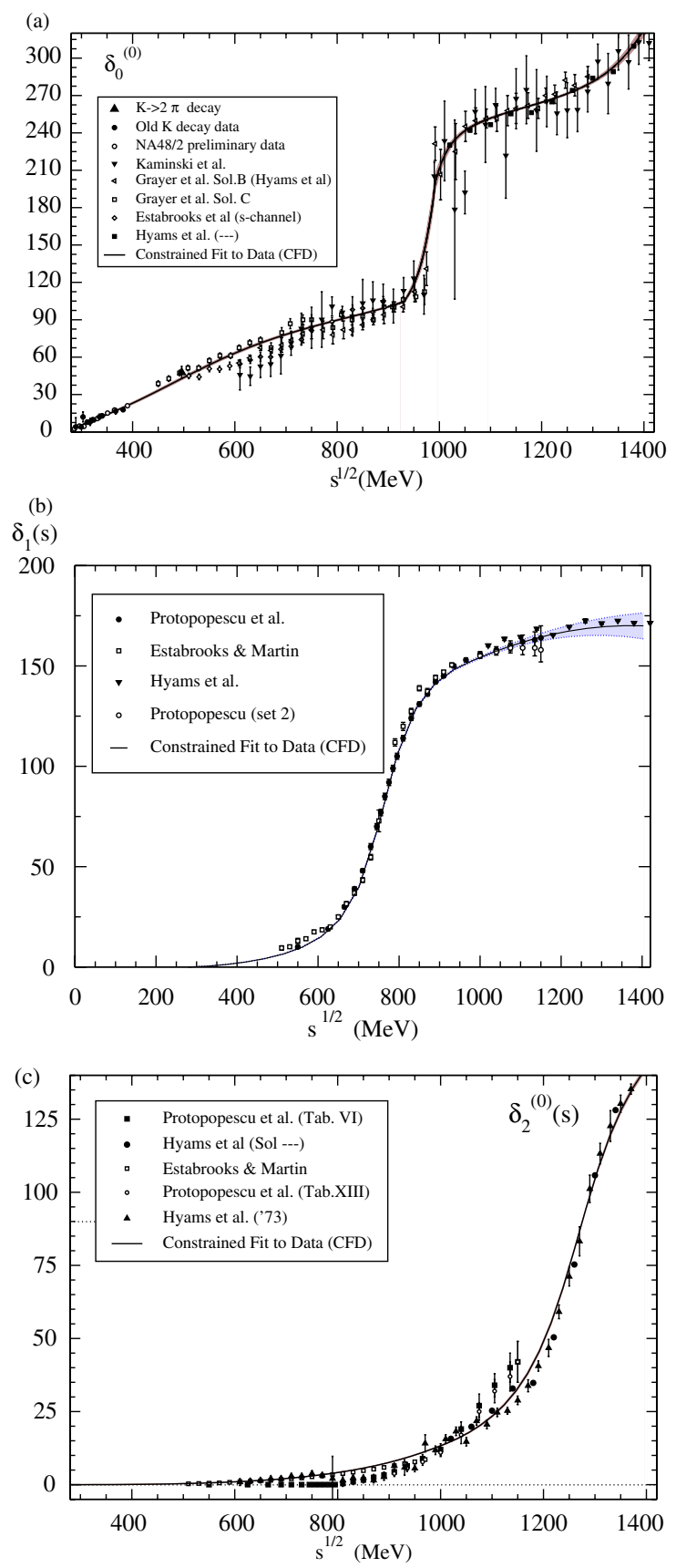

FIG. 13 (color online). (a) S0 wave phase shift (CFD set). Some data from Refs. [4,6] are also shown. Notice the hump around $700 \mathrm{MeV}$. (b) P wave phase shift (CFD set); errors below $1 \mathrm{GeV}$ are as the thickness of the line. Some data from Refs. $[4,6]$ are also shown. Note, however, that $\delta_{1}$ is obtained fitting the $\pi \pi$ scattering data only above $\bar{K} K$ threshold; below it, it is obtained fitting the pion form factor (cf. Ref. [8]). (c) D0 wave phase shift (CFD set); errors as the thickness of the line. Some data from Refs. [4,6] are also shown.

this same limit. The S0, P, and D0 phase shifts, with the values of the CFD set, are shown in Fig. 13.

In connection with the S0 wave, there is some interest attached to the location of the so-called " $\sigma$ pole." This has been discussed in Ref. [3]; we there found, with the pa- 
rameters of the UFD set,

$$
\begin{aligned}
M_{\sigma} & =484 \pm 6(\text { St. }) \pm 11 \text { (Sys. }) \mathrm{MeV}, \\
\Gamma_{\sigma} / 2 & =255 \pm 8(\text { St. }) \pm 2(\text { Sys. }) \mathrm{MeV} .
\end{aligned}
$$

For the CFD set we now have

$$
\begin{aligned}
M_{\sigma} & =473 \pm 6(\text { St. }) \pm 11 \text { (Sys. }) \mathrm{MeV} \\
\Gamma_{\sigma} / 2 & =257 \pm 5(\text { St. }) \pm 2 \text { (Sys. }) \mathrm{MeV} .
\end{aligned}
$$

Here the first error is the statistical one, due to the errors in the parameters of the S0 wave, and the second the error induced by the extrapolation, as estimated in Ref. [3]. We gave the numbers not taking into account isospin breaking; if we took it into account (see below for a discussion of this), the central values would change a little, to $M_{\sigma}=478$ and $\Gamma_{\sigma} / 2=242$ for the CFD set.

Nevertheless, we must remark that a really precise determination of the location of this resonance requires the use of the Roy equations. We will present this in a future paper.

The S2 wave is not found with a precision similar to that of the S0 or P waves. This is, of course, due to the lack of accuracy of the experimental data on states with isospin two (but, on the other hand, the corresponding scattering length, $a_{0}^{(2)}$, is found with good accuracy). The lack of experimental accuracy is what prevents more precise values for the D2 wave, and something similar happens for the $\mathrm{F}$ wave.

By comparison with an analysis similar to ours, our values for S0, D waves are a factor of 3 or four more precise than those in Ref. [13]. Our D2 wave, uncertain as it is, is still much more reliable than what is given in Ref. [13]: where a parametrization for this wave is given that is incompatible with their own findings at low energy (the scattering length), and with Regge theory and experimental data at high energy. Finally, at high energy (in the Regge region), our amplitudes fit well the existing data, something that the amplitudes in Ref. [13] fail to do by a factor of 2 .

We have also used these scattering amplitudes to evaluate low energy parameters for P, D0, D2, and F waves, and other observables, clearly improving on previous work. These parameters may then be used to test chiral perturbation theory to one and two loops, or to find quantities relevant for $\mathrm{CP}$ violating kaon decays. In particular, for the S-wave scattering lengths we find the very accurate determinations

$$
\begin{aligned}
& a_{0}^{(0)}=0.223 \pm 0.009 M_{\pi}^{-1}, \\
& a_{0}^{(2)}=-0.0444 \pm 0.0045 M_{\pi}^{-1} .
\end{aligned}
$$

This can be compared with what is found in Ref. [17], using chiral perturbation theory with Roy equations and experimental data input,

$$
\begin{aligned}
& a_{0}^{(0)}=0.220 \pm 0.005 M_{\pi}^{-1}, \\
& a_{0}^{(2)}=-0.0444 \pm 0.0010 M_{\pi}^{-1} .
\end{aligned}
$$

that is, a remarkable agreement at very low energies. However, at higher energies our S0 wave phase shift deviates somewhat from that obtained in Ref. [17]. This deviation is due to the hump we find in the 400 to $900 \mathrm{MeV}$ region, that makes our phase shifts larger than those of Ref. [17]. [It is to be noted, on the other hand, that this hump is also generated naturally within the framework of unitarized chiral perturbation theory, Ref. [18], when fitting all existing scattering data (using a large systematic error to cover all phase shift sets)]. The S2 wave also deviates slightly (but significantly) from that of Ref. [17] above $500 \mathrm{MeV}$. In addition our analysis has other marked differences compared to that of Ref. [17], mainly in the D waves and Regge description at high energies, that have already been explained in detail in Ref. [19].

One can ask the question whether it would be possible to improve on our precision. The answer is, no in the sense that our amplitudes agree, within errors, with theoretical requirements and with data. ${ }^{9}$ Moreover, for some waves (notably, S0, P, and D0, shown in Fig. 13) our precision is at the limit of the estimated corrections due to breaking of isospin invariance. For other waves, known less precisely, a sizable improvement would require substantially improved experimental data; certainly for the S2 wave at low energy, and for all the waves above $\sim 1 \mathrm{GeV}$. This is particularly true for the inelasticities, very poorly determined from experiment, except for the S0 wave where the lucky coincidence that it is mostly given by $\bar{K} K$ states and the existence of data on the process $\pi \pi \rightarrow \bar{K} K$ saves the day. It may be assumed that, perhaps, imposing exact fulfillment of FDR and Roy equations could improve our errors. We have found this impossible: increasing the weight of FDR and Roy equations in the CFD, Eq. (5.4), results in amplitudes that indeed satisfy better (but not that much better) FDR and Roy equations, but that deviate from experiment by intolerable amounts. This is not surprising; there are important features that fail to be resolved by existing experimental analyses. For example, we have the matter of the inelasticity of the D0 wave near $1 \mathrm{GeV}$, or the lack of information on the $\rho(1450)$ resonance, clearly seen in $e^{+} e^{-}$annihilations but for which the particle data tables refrain from giving the $2 \pi$ branching ratio-and which is absent from our analysis. This could give a sizable contribution above $1380 \mathrm{MeV}$ : the absence of the $\rho(1450)$ resonance in our $\mathrm{P}$ wave is likely responsible for the structure found in $\Delta_{0+}, \Delta_{1}$ above $\sim 1 \mathrm{GeV}$; cf. Figs. 5

\footnotetext{
${ }^{9}$ One may think that imposing chiral perturbation theory could lead to decreasing the errors of the $\pi \pi$ scattering amplitudes. However, this matter is complicated and is left for a future publication: at least the analysis can be now made from the well grounded set of pion-pion amplitudes given by our fits.
} 
and 8. Unfortunately, taking this $\rho(1450)$ resonance into account correctly requires a multichannel calculation, which, even if possible, lies outside the scope of the present article.

As for the S0 wave, the contribution of $4 \pi$ states, dominant above $\sim 1350 \mathrm{MeV}$, is not properly taken into account. Likewise, our Regge formulas only give the imaginary parts of the scattering amplitudes in the mean, in the energy region $1420 \mathrm{MeV} \leq s^{1 / 2} \leqslant 1800 \mathrm{MeV}$; a region where neither phase shift analyses nor Regge fits give precise results: our scattering amplitudes are not well determined in the region from 1350 to $1800 \mathrm{MeV}$. Until much better experimental data, in particular, on phase shifts and (above all) inelasticities above $\bar{K} K$ thresholds are available, the set CFD of pion-pion amplitudes will remain the best that one can do, from experiment. ${ }^{10}$

Next, we say a few words with respect to isospin breaking corrections, neglected in our analysis up till now. In most cases, our errors are sufficiently large to cover the estimated values of such effects; but there are two exceptions. For the $\mathrm{P}$ wave, our errors include isospin breaking corrections. This is possible because we can evaluate this wave either from the pion form factor in $e^{+}+e^{-} \rightarrow \pi^{+}+$ $\pi^{-}$or in $\tau \rightarrow \nu+\pi^{+}+\pi^{0}$; see Ref. [8] for the details. And a special case is the $\mathrm{S} 0$ wave. Here a recent calculation has been made[20] in which account is taken, for Ke4 decays, of the fact that in the real world isospin is broken. According to Ref. [20], this is done by subtracting, from the experimental phase shift, as given in Ref. [4], the correction

$$
\begin{aligned}
\Delta \delta_{0}^{(0)}= & \frac{1}{32 \pi F_{\pi}^{2}}\left\{\left[4\left(M_{\pi}^{2}-M_{\pi^{0}}^{2}\right)+s\right] \beta(s)\right. \\
& \left.+\left(s-M_{\pi^{0}}^{2}\right)\left[1+\frac{3}{2} r\right] \beta_{0}(s)\right\}
\end{aligned}
$$

where $F_{\pi} \simeq 92.6 \mathrm{MeV}$ is the pion decay constant, $M_{\pi^{0}}$ is the mass of the neutral pion, $\beta(s)=\sqrt{1-4 M_{\pi}^{2} / s}$, $\beta_{0}(s)=\sqrt{1-4 M_{\pi^{0}}^{2} / s}, \quad$ and $\quad r=\left(m_{d}-m_{u}\right) /\left(m_{s}-\right.$ $\left.m_{d} / 2-m_{u} / 2\right)$.

If we take the results of this calculation at face value, we can repeat our fits taking it into account. For the UFD set, only the low energy S0 wave is affected, and it is so very little: by less than $1^{\circ}$ above $400 \mathrm{MeV}$ [which is not surprising, since, in fact, the correction (7.3) is actually somewhat smaller than the experimental errors over most of the range]. Only the scattering length moves beyond 1 standard deviation, to $a_{0}^{(0)}=0.210 \pm 0.010$. For the CFD set, the changes induced by incorporation of isospin break-

\footnotetext{
${ }^{10}$ That the poor information above $\bar{K} K$ threshold is responsible for the limitations in the fulfillment of dispersion relations is seen if we realize that FDR are satisfied to an average $\bar{d}_{\mathrm{FDR}}^{2}=$ 0.49 below $932 \mathrm{MeV}$, and Roy equations are satisfied to an average $\bar{d}_{\text {Roy }}^{2}=0.17$ below the $\bar{K} K$ threshold.
}

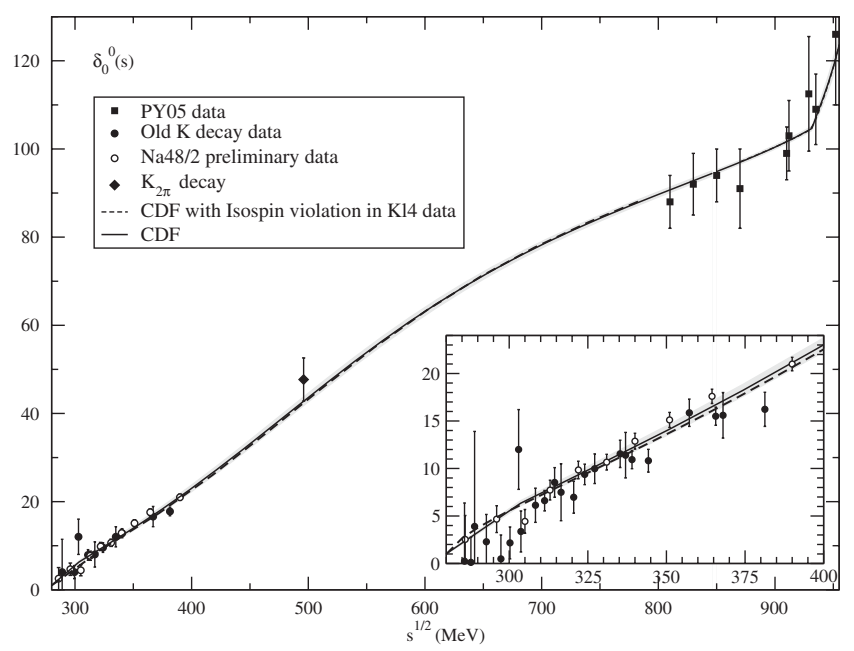

FIG. 14. S0 wave phase shift (CFD set): continuous line, neglecting isospin corrections; broken line, with isospin corrections taken into account. Notice that the hump around $700 \mathrm{MeV}$ is basically identical for both. Some data from Refs. [4,6] are also shown.

ing corrections in the waves other than the S0 wave are almost negligible. The S0 wave moves closer to the one given in the present paper, from which it is almost indistinguishable (Fig. 14); and the scattering length becomes compatible with what we found neglecting isospin breaking: we now get $a_{0}^{(0)}=0.213 \pm 0.011$. Indeed, including isospin breaking corrections does not much affect our results. The corresponding values of the parameters for the S0 wave, the only ones that change appreciably, are given in Appendices A and B; a comparison between what one finds with/without isospin corrections for the S0 phase shift can be seen, with difficulty (because they are so close one to the other) in Fig. 14.

We finish with a comment regarding the relative merits of sets UFD and CFD. It is clear that the central values of the CFD set should be considered as the preferred ones since they incorporate fulfillment of analyticity requirements: analyticity of the individual waves themselves (for the low energy region), as well as analyticity and crossing symmetry of the scattering amplitudes in the form of forward dispersion relations, sum rules, and Roy equations. However, the price to pay for the last two requirements is that all waves are now correlated: in this sense, the UFD set is more robust. Since both solutions fit data, and are very similar, one can use one or the other almost interchangeably, except for S2 and, above all, D2 waves, for which the CFD set is clearly superior.

\section{ACKNOWLEDGMENTS}

F. J. Y.'s work was supported in part by the Spanish DGI of the MEC under Contract No. FPA2003-04597. J. R. P.'s research is partially funded by Spanish CICYT Contracts No. FPA2005-02327 and No. BFM2003-00856 as well as 
Banco Santander/Complutense Contract No. PR27/05$13955-\mathrm{BSCH}$, and is part of the EU integrated infrastructure initiative HADRONPHYSICS PROJECT, under Contract No. RII3-CT-2004-506078. We are grateful to H. Leutwyler for his remarks about the $\bar{K} K$ threshold rise of the D0 wave and R. García Martín for information concerning the location of the $\sigma$ pole. R. K. thanks the Universidad Autónoma de Madrid and Universidad Complutense de Madrid, where part of this research was carried out.

\section{APPENDIX A: FITS TO DATA UP TO $1.42 \mathrm{GEV}$ (UFD SET)}

In this appendix we collect the best values for the parametrizations of the various partial waves obtained by fitting experimental data, with the procedures defined in Refs. [1,2]; see also Sec. II here.

\section{The S0 wave}

The region $s^{1 / 2} \leq 932 \mathrm{GeV}$. We take $s_{0}=4 m_{K}^{2}$, and take the Adler zero at $s=\frac{1}{2} z_{0}^{2}$, with $z_{0}$ fixed at $M_{\pi}$. We find

$$
\begin{aligned}
\cot \delta_{0}^{(0)}(s)= & \frac{s^{1 / 2}}{2 k s-\frac{1}{2} z_{0}^{2}}\left\{\frac{z_{0}^{2}}{M_{\pi} \sqrt{s}}+B_{0}+B_{1} \frac{\sqrt{s}-\sqrt{s_{0}}-s}{\sqrt{s}+\sqrt{s_{0}}-s}\right. \\
& \left.+B_{2}\left[\frac{\sqrt{s}-\sqrt{s_{0}-s}}{\sqrt{s}+\sqrt{s_{0}-s}}\right]^{2}\right\}, \\
z_{0} \equiv & M_{\pi} ; B_{0}=4.3 \pm 0.3, \\
B_{1}= & -26.7 \pm 0.6, \\
B_{2}= & -14.1 \pm 1.4 .
\end{aligned}
$$

This corresponds to neglect of isospin breaking. If isospin breaking is taken into account as in Ref. [20], we find instead

$$
\begin{gathered}
B_{0}=3.80 \pm 0.34, \quad B_{1}=-27.1 \pm 0.8, \\
B_{2}=-8.3 \pm 1.8 ; \quad a_{0}^{(0)}=0.211 \pm 0.010 M_{\pi}^{-1}, \\
b_{0}^{(0)}=0.278 \pm 0.010 M_{\pi}^{-3} .
\end{gathered}
$$

The SO wave between $932 \mathrm{MeV}$ and $1420 \mathrm{MeV}$. We here use the K-matrix fit of Ref. [2]. ${ }^{11}$

$$
\tan \delta_{0}^{(0)}(s)= \begin{cases}\frac{k_{1}\left|k_{2}\right| \operatorname{det} \mathbf{K}+k_{1} K_{11}}{1+\left|k_{2}\right| K_{22}}, & s \leq 4 m_{K}^{2}, \\ \frac{1}{2 k_{1}\left[K_{11}+k_{2}^{2} K_{22} \operatorname{det} \mathbf{K}\right]}\left\{k_{1}^{2} K_{11}^{2}-k_{2}^{2} K_{22}^{2}+k_{1}^{2} k_{2}^{2}(\operatorname{det} \mathbf{K})^{2}-1\right. & \\ +\sqrt{\left.\left(k_{1}^{2} K_{11}^{2}+k_{2}^{2} K_{22}^{2}+k_{1}^{2} k_{2}^{2}(\operatorname{det} \mathbf{K})^{2}+1\right)^{2}-4 k_{1}^{2} k_{2}^{2} K_{12}^{4}\right\},} & s \geq 4 m_{K}^{2}\end{cases}
$$

and

$$
\eta_{0}^{(0)}(s)=\sqrt{\frac{\left(1+k_{1} k_{2} \operatorname{det} \mathbf{K}\right)^{2}+\left(k_{1} K_{11}-k_{2} K_{22}\right)^{2}}{\left(1-k_{1} k_{2} \operatorname{det} \mathbf{K}\right)^{2}+\left(k_{1} K_{11}+k_{2} K_{22}\right)^{2}}}, \quad s \geq 4 m_{K}^{2} .
$$

Here $k_{1}=\frac{1}{2} \sqrt{s-4 M_{\pi}^{2}}, k_{2}=\frac{1}{2} \sqrt{s-4 m_{K}^{2}}$ and the K-matrix elements are

$$
K_{i j}(s)=\frac{\mu \alpha_{i} \alpha_{j}}{M_{1}^{2}-s}+\frac{\mu \beta_{i} \beta_{j}}{M_{2}^{2}-s}+\frac{1}{\mu} \gamma_{i j} .
$$

$\mu$ is a mass scale, that we take $\mu=1 \mathrm{GeV}$. The powers of $\mu$ have been arranged so that the $\alpha_{i}, \beta_{i}, \gamma_{i j}$ are dimensionless; they are also assumed to be constant. The pole at $M_{1}^{2}$ simulates the left-hand cut of $\mathbf{K}$, and the pole at $M_{2}^{2}$ is connected with the phase shift crossing $270^{\circ}$ around $1.3 \mathrm{GeV}$; both poles are necessary to get a good fit. The values of the parameters are

\footnotetext{
${ }^{11}$ A polynomial fit, giving results very similar to the K-matrix fit, can also be given. It may be found in Appendix B of Ref. [2].
}

$$
\begin{gathered}
\alpha_{1}=0.843 \pm 0.017, \quad \alpha_{2}=0.20 \pm 0.06, \\
\beta_{1}=1.02 \pm 0.02 \quad \beta_{2}=1.33 \pm 0.013, \\
\gamma_{11}=3.10 \pm 0.11, \quad \gamma_{12}=1.82 \pm 0.05 \\
\gamma_{22}=-7.00 \pm 0.04, \quad M_{1}=0.888 \pm 0.004 \mathrm{GeV} \\
M_{2}=1.327 \pm 0.004 \mathrm{GeV} .
\end{gathered}
$$

$M_{1}$ lies near the beginning of the left-hand cut for $\bar{K} K \rightarrow$ $\pi \pi$ scattering, located at $0.952 \mathrm{GeV}$. The parameters in (A2c) are strongly correlated. In fact, we have verified that there exists a wide set of minima, with very different values of the parameters. Nevertheless, the corresponding values of $\delta_{0}^{(0)}$ and $\eta_{0}^{(0)}$ vary very little in all these minima, so that (A2c) can be considered a faithful representation of the S0 wave for $\pi \pi$ scattering. The representations of the S0 wave are matched exactly at $932 \mathrm{MeV}$, where one has

$$
\delta_{0}^{(0)}\left((932 \mathrm{MeV})^{2}\right)=104.9 \pm 0.5^{\circ} .
$$

\section{Parametrization of the $S 2$ wave}

The region $s^{1 / 2} \leq 932 \mathrm{GeV}$. For isospin 2, there is no low energy resonance, but this wave presents the feature that a zero is expected (and, indeed, confirmed by the fits). 
It is related to the so-called Adler zeros; to lowest order in chiral perturbation theory, one has the zero at $s=2 z_{2}^{2}$, with $z_{2}=M_{\pi}$. We note that, unlike the corresponding zero for the S0 wave, $2 z_{2}^{2}$ is inside the region where the conformal expansion is expected to converge well. We here fix $z_{2}=$ $M_{\pi}$ and write

$$
\begin{aligned}
\cot \delta_{0}^{(2)}(s) & =\frac{s^{1 / 2} M_{\pi}^{2}}{2 k s-2 z_{2}^{2}}\left\{B_{0}+B_{1} \frac{\sqrt{s}-\sqrt{s_{l}-s}}{\sqrt{s}+\sqrt{s_{l}-s}}\right\}, \\
z_{2} & \equiv M_{\pi}, \quad s_{l}^{1 / 2}=1.05 \mathrm{GeV} .
\end{aligned}
$$

Then we get

$$
B_{0}=-80.4 \pm 2.8, \quad B_{1}=-73.6 \pm 10.5 .
$$

The S2 wave between $932 \mathrm{MeV}$ and $1420 \mathrm{MeV}$. We require a junction with the low energy phase shift, and its derivative, at $932 \mathrm{MeV}$, neglect inelasticity for the phase shift below $1.45 \mathrm{GeV}$, and write

$$
\begin{aligned}
\cot \delta_{0}^{(2)}(s)= & \frac{s^{1 / 2} M_{\pi}^{2}}{2 k s-2 M_{\pi}^{2}}\left\{B_{h 0}+B_{h 1}\left[w_{h}(s)-w_{h}\left(s_{M}\right)\right]\right. \\
& \left.+B_{h 2}\left[w_{h}(s)-w_{h}\left(s_{M}\right)\right]^{2}\right\}, \\
s^{1 / 2} \geq & 932 \mathrm{MeV}, \quad B_{h 0}=B_{0}+B_{1} w_{l}\left(s_{M}\right), \\
B_{h 1}= & \left.B_{1} \frac{\partial w_{l}(s)}{\partial w_{h}(s)}\right|_{s=s_{M}} ; \\
w_{l}(s)= & \frac{\sqrt{s}-\sqrt{s_{l}-s}}{\sqrt{s}+\sqrt{s_{l}-s}}, \quad s_{l}=(1050 \mathrm{MeV})^{2}, \\
w_{h}(s)= & \frac{\sqrt{s}-\sqrt{s_{h}}-s}{\sqrt{s}+\sqrt{s_{h}-s}}, \quad s_{h}=(1450 \mathrm{MeV})^{2} .
\end{aligned}
$$

$B_{h 2}$ is a free parameter. We get

$$
B_{h 2}=112 \pm 38 \text {. }
$$

The inelasticity is described by the empirical fit

$$
\begin{aligned}
& \eta_{0}^{(2)}(s)=1-\epsilon\left(1-s_{l} / s\right)^{3 / 2}, \\
\epsilon= & 0.17 \pm 0.12 \quad\left(s_{l}^{1 / 2}=1.05 \mathrm{GeV}\right) .
\end{aligned}
$$

\section{The $P$ wave}

The region $s^{1 / 2} \leq 2 m_{K}$. We have

$$
\begin{aligned}
\cot \delta_{1}(s) & =\frac{s^{1 / 2}}{2 k^{3}}\left(M_{\rho}^{2}-s\right)\left\{\frac{2 M_{\pi}^{3}}{M_{\rho}^{2} \sqrt{s}}+B_{0}+B_{1} \frac{\sqrt{s}-\sqrt{s_{0}}-\bar{s}}{\sqrt{s}+\sqrt{s_{0}}-\bar{s}}\right\}, \\
s_{0}^{1 / 2} & =1.05 \mathrm{GeV} .
\end{aligned}
$$

The best result is

$$
\begin{gathered}
B_{0}=1.055 \pm 0.011, \quad B_{1}=0.15 \pm 0.05, \\
M_{\rho}=773.6 \pm 0.9 \mathrm{MeV} .
\end{gathered}
$$

The $P$ wave between $2 m_{K}$ and $1420 \mathrm{MeV}$. We use a purely phenomenological parametrization:

$$
\begin{aligned}
\delta_{1}(s) & =\lambda_{0}+\lambda_{1}\left(\sqrt{s / 4 m_{K}^{2}}-1\right)+\lambda_{2}\left(\sqrt{s / 4 m_{K}^{2}}-1\right)^{2}, \\
\eta_{1}(s) & =1-\epsilon_{1} \sqrt{1-4 m_{K}^{2} / s}-\epsilon_{2}\left(1-4 m_{K}^{2} / s\right), \\
s & >4 m_{K}^{2} .
\end{aligned}
$$

The phase at the low energy edge, $\delta_{1}\left((0.992 \mathrm{GeV})^{2}\right)=$ $153.63 \pm 0.55^{\circ}$, is obtained from the fit at low energy above; this fixes $\lambda_{0}$. The rest of the parameters follow from the fit at intermediate energy. We have

$$
\begin{gathered}
\lambda_{0}=2.681 \pm 0.010, \quad \lambda_{1}=1.57 \pm 0.18, \\
\lambda_{2}=-1.96 \pm 0.49, \quad \epsilon_{1}=0.10 \pm 0.06, \\
\epsilon_{2}=0.11 \pm 0.11 .
\end{gathered}
$$

\section{Parametrization of the Do wave}

The region $s^{1 / 2} \leq 2 m_{K}$. To take into account the analyticity structure, we fit with different expressions for energies below and above the $\bar{K} K$ threshold, requiring however exact matching at $s=4 m_{K}^{2}$. Below the $\bar{K} K$ threshold we take into account the existence of nonnegligible inelasticity above $1.05 \mathrm{GeV}$, which is near the $\rho \pi \pi$ threshold, by choosing a conformal variable $w$ appropriate to a plane cut for $s>(1.05 \mathrm{GeV})^{2}$. So we write

$$
\begin{aligned}
\cot \delta_{2}^{(0)}(s) & =\frac{s^{1 / 2}}{2 k^{5}}\left(M_{f_{2}}^{2}-s\right) M_{\pi}^{2}\left\{B_{0}+B_{1} w\right\}, \quad s<4 m_{K}^{2}, \\
w & =\frac{\sqrt{s}-\sqrt{s_{0}}-s}{\sqrt{s}+\sqrt{s_{0}-s}}, \quad s_{0}^{1 / 2}=1.05 \mathrm{GeV} . \quad \text { (A7a) }
\end{aligned}
$$

The mass of the $f_{2}$ we fix at $M_{f_{2}}=1275.4 \mathrm{MeV}$; no error is taken for this quantity, since it is negligibly small $(1.2 \mathrm{MeV})$ when compared with the other errors. We find the values of the parameters

$$
B_{0}=12.47 \pm 0.12, \quad B_{1}=10.12 \pm 0.16 .
$$

The DO wave between $2 m_{K}$ and $1420 \mathrm{MeV}$. Above the $\bar{K} K$ threshold we use the following formula for the phase shift:

$$
\begin{gathered}
\cot \delta_{2}^{(0)}(s)=\frac{s^{1 / 2}}{2 k^{5}}\left(M_{f_{2}}^{2}-s\right) M_{\pi}^{2}\left\{B_{h 0}+B_{h 1} w\right\}, \\
s>4 m_{K}^{2}, \quad w=\frac{\sqrt{s}-\sqrt{s_{h}-s}}{\sqrt{s}+\sqrt{s_{h}-s}}, \quad s_{h}^{1 / 2}=1.45 \mathrm{GeV} .
\end{gathered}
$$

This neglects inelasticity below $1.45 \mathrm{GeV}$, which is approximately the $\rho \rho$ threshold; inelasticity will be added by hand; see below. We require exact matching with the low energy expression, which yields the value of $B_{h 0} . B_{h 1}$ follows from the fit at intermediate energy. We find 


$$
B_{h 0}=18.77 \pm 0.16, \quad B_{h 1}=43.7 \pm 1.8 .
$$

For the inelasticity we write, as discussed in the main text,

$$
\eta_{2}^{(0)}(s)=\left\{\begin{array}{l}
1, \quad s<4 m_{K}^{2}, \\
1-\epsilon\left(1-\frac{4 m_{K}^{2}}{s}\right)^{5 / 2}\left(1-\frac{4 m_{K}^{2}}{M_{f_{2}}^{2}}\right)^{-5 / 2}\left\{1+r\left[1-\frac{k_{2}(s)}{k_{2}\left(M_{f_{2}}^{2}\right)}\right]\right\}, \quad \epsilon=0.284 \pm 0.030, \quad r=2.54 \pm 0.31 . \\
s>4 m_{K}^{2}, \quad k_{2}=\sqrt{s / 4-m_{K}^{2}} .
\end{array}\right.
$$

\section{Parametrization of the D2 wave}

For isospin equal 2, there are no resonances in the $\mathrm{D}$ wave. If we want a parametrization that applies down to threshold, we must incorporate the zero of the corresponding phase shift. We write

$$
\begin{aligned}
& \cot \delta_{2}^{(2)}(s)= \frac{s^{1 / 2}}{2 k^{5}}\left\{B_{0}+B_{1} w(s)\right. \\
&\left.+B_{2} w(s)^{2}\right\} \frac{M_{\pi}^{4} s}{4\left(M_{\pi}^{2}+\Delta^{2}\right)-s}, \\
& s^{1 / 2} \leq 1.05 \mathrm{GeV},
\end{aligned}
$$

with $\Delta$ a free parameter and

$$
w(s)=\frac{\sqrt{s}-\sqrt{s_{0}-s}}{\sqrt{s}+\sqrt{s_{0}-s}}, \quad s_{0}^{1 / 2}=1450 \mathrm{MeV} .
$$

Moreover, we impose the value for the scattering length that follows from the Froissart-Gribov representation. We find

$$
\begin{array}{ll}
B_{0}=(2.4 \pm 0.5) \times 10^{3}, & B_{1}=(7.8 \pm 1.0) \times 10^{3}, \\
B_{2}=(23.7 \pm 4.2) \times 10^{3}, & \Delta=196 \pm 25 \mathrm{MeV} .
\end{array}
$$

For the inelasticity, above $1.05 \mathrm{GeV}$,

$$
\begin{gathered}
\eta_{2}^{(2)}(s)=1-\epsilon(1-\hat{s} / s)^{3}, \quad \hat{s}^{1 / 2}=1.05 \mathrm{GeV}, \\
\epsilon=0.2 \pm 0.2 ;
\end{gathered}
$$

this is negligible up to $1.25 \mathrm{GeV}$.

\section{The $F$ wave}

For the $\mathrm{F}$ wave below $s^{1 / 2}=1.42 \mathrm{GeV}$ we fit the experimental phase shifts plus the scattering length as given by the Froissart-Gribov representation. We have

$$
\begin{aligned}
\cot \delta_{3}(s) & =\frac{s^{1 / 2}}{2 k^{7}} M_{\pi}^{6}\left\{\frac{2 \lambda M_{\pi}}{\sqrt{s}}+B_{0}+B_{1} \frac{\sqrt{s}-\sqrt{s_{0}-s}}{\sqrt{s}+\sqrt{s_{0}}-s}\right\}, \\
s_{0}^{1 / 2} & =1.45 \mathrm{GeV}, \quad B_{0}=(1.09 \pm 0.03) \times 10^{5}, \\
B_{1} & =(1.41 \pm 0.04) \times 10^{5}, \quad \lambda=0.051 \times 10^{5} .
\end{aligned}
$$

We neglect the inelasticity of the $\mathrm{F}$ wave below $1.45 \mathrm{GeV}$. The contribution of the $\mathrm{F}$ wave to all our sum rules is very small (but not always negligible); the interest of calculating it lies in that it provides a test (by its very smallness) of the convergence of the partial wave expansions.

\section{The $\mathbf{G}$ waves}

For the G0 wave, we take its imaginary part to be given by

$$
\begin{aligned}
\operatorname{Im} \hat{f}_{4}^{(0)}(s)= & \left(\frac{k(s)}{k\left(M_{f_{4}}^{2}\right)}\right)^{18} \\
& \times \mathrm{BR} \frac{M_{f_{4}}^{2} \Gamma^{2} \mathrm{e}^{2 c\left(1-s / M_{f_{4}}^{2}\right)^{2}}}{\left(s-M_{f_{4}}^{2}\right)^{2}+M_{f_{4}}^{2} \Gamma^{2}\left[k(s) / k\left(M_{f_{4}}^{2}\right)\right]^{18}}, \\
\mathrm{BR}= & 0.17 \pm 0.02, \quad M_{f_{4}}=2025 \pm 8 \mathrm{MeV}, \\
\Gamma= & 194 \pm 13 \mathrm{MeV}, \quad c=9.23 \pm 0.46 . \quad \text { (A11) }
\end{aligned}
$$

For the wave G2, we can write, neglecting its eventual inelasticity,

$$
\begin{gathered}
\cot \delta_{4}^{(2)}(s)=\frac{s^{1 / 2} M_{\pi}^{8}}{2 k^{9}} B, \quad B=(-9.1 \pm 3.3) \times 10^{6}, \\
s^{1 / 2} \geq 1 \mathrm{GeV} .
\end{gathered}
$$

It should be noted that the expressions for the G0, G2 waves, are little more than order of magnitude estimates. Moreover, at low energies the expression for G2 certainly fails; below $1 \mathrm{GeV}$, an expression in terms of the scattering length approximation, with

$$
a_{4}^{(2)}=(4.5 \pm 0.2) \times 10^{-6} M_{\pi}^{-9},
$$

is more appropriate.

\section{APPENDIX B: FITS UP TO 1.42 GEV, IMPROVED WITH DISPERSION RELATIONS (CFD SET)}

In this appendix we collect the best values for the parametrizations of the various partial waves, after improving with the help of dispersion relations: forward dispersion relations and Roy equations. In addition, we required verification (within errors) of the two crossing sum rules in Sec. IV. 
All the formulas are as before improvement, i.e., as in Appendix A; only the central values of the parameters change. We give in some detail only the S0 and S2 waves, because now we allow variation of the location of the Adler zeros. For the other waves, all the formulas are exactly as in Appendix A.

\section{The S0 wave}

The region $s^{1 / 2} \leq 932 \mathrm{GeV}$. We take $s_{0}=4 m_{K}^{2}$, and impose the Adler zero at $s=\frac{1}{2} z_{0}^{2}$, with $z_{0}$ free. We find

$$
\begin{aligned}
\cot \delta_{0}^{(0)}(s)= & \frac{s^{1 / 2}}{2 k s-\frac{1}{2} z_{0}^{2}}\left\{\frac{z_{0}^{2}}{M_{\pi} \sqrt{s}}+B_{0}+B_{1} \frac{\sqrt{s}-\sqrt{s_{0}-s}}{\sqrt{s}+\sqrt{s_{0}-\bar{s}}}\right. \\
& \left.+B_{2}\left[\frac{\sqrt{s}-\sqrt{s_{0}-s}}{\sqrt{s}+\sqrt{s_{0}-\bar{s}}}\right]^{2}\right\}, \\
B_{0}= & 4.41 \pm 0.30, \quad B_{1}=-26.25 \pm 0.60, \\
B_{2}= & -15.8 \pm 1.4, \quad z_{0}=166.1 \pm 4.2 \mathrm{MeV} .
\end{aligned}
$$

This corresponds to neglect of isospin breaking. If isospin breaking is taken into account as in Ref. [20], we find instead

$$
\begin{array}{ll}
B_{0}=3.93 \pm 0.34, & B_{1}=-26.84 \pm 0.78 \\
B_{2}=-10.5 \pm 1.8, & z_{0}=150.2 \pm 4.2 \mathrm{MeV}
\end{array}
$$

The SO wave between $932 \mathrm{MeV}$ and $1420 \mathrm{MeV}$. The $\mathrm{K}$ matrix parameters are almost unchanged; we have now

$$
\begin{gathered}
\alpha_{1}=0.843 \pm 0.017, \quad \alpha_{2}=0.20 \pm 0.06 \\
\beta_{1}=1.02 \pm 0.02, \quad \beta_{2}=1.33 \pm 0.013 \\
\gamma_{11}=3.10 \pm 0.11, \quad \gamma_{12}=1.81 \pm 0.05 \\
\gamma_{22}=-7.00 \pm 0.04, \quad M_{1}=0.888 \pm 0.004 \mathrm{GeV} \\
M_{2}=1.327 \pm 0.004 \mathrm{GeV}
\end{gathered}
$$

\section{The $\mathrm{S} 2$ wave}

The region $s^{1 / 2} \leq 932 \mathrm{MeV}$. We here leave $z_{2}$ free. Then,

$$
\begin{array}{rlrl}
\cot \delta_{0}^{(2)}(s) & =\frac{s^{1 / 2} M_{\pi}^{2}}{2 k s-2 z_{2}^{2}}\left\{B_{0}+B_{1} \frac{\sqrt{s}-\sqrt{s_{l}-s}}{\sqrt{s}+\sqrt{s_{l}-s}}\right\}, \quad s_{l}^{1 / 2}=1.05 \mathrm{GeV} \\
B_{0} & =-80.2 \pm 2.8, \quad B_{1}=-69.4 \pm 10.5, & z_{2} & =145.0 \pm 3.6 \mathrm{MeV}
\end{array}
$$

The S2 wave between $932 \mathrm{MeV}$ and $1420 \mathrm{MeV}$. We write

$$
\begin{aligned}
\cot \delta_{0}^{(2)}(s)= & \frac{s^{1 / 2} M_{\pi}^{2}}{2 k s-2 z_{2}^{2}}\left\{B_{h 0}+B_{h 1}\left[w_{h}(s)-w_{h}\left(s_{M}\right)\right]\right. \\
& \left.+B_{h 2}\left[w_{h}(s)-w_{h}\left(s_{M}\right)\right]^{2}\right\}, \\
s^{1 / 2} \geq & 932 \mathrm{MeV}, \quad B_{h 0}=B_{0}+B_{1} w_{l}\left(s_{M}\right), \\
B_{h 1}= & \left.B_{1} \frac{\partial w_{l}(s)}{\partial w_{h}(s)}\right|_{s=s_{M}} ;
\end{aligned}
$$

$z_{2}$ is given in $(\mathrm{B} 3 \mathrm{a})$;

$$
\begin{array}{ll}
w_{l}(s)=\frac{\sqrt{s}-\sqrt{s_{l}-s}}{\sqrt{s}+\sqrt{s_{l}-s}}, & s_{l}=(1050 \mathrm{MeV})^{2} \\
w_{h}(s)=\frac{\sqrt{s}-\sqrt{s_{h}-s}}{\sqrt{s}+\sqrt{s_{h}-s}}, & s_{h}=(1450 \mathrm{MeV})^{2} .
\end{array}
$$

We get

The inelasticity is $B_{h 2}=120 \pm 38$.

$$
\begin{gathered}
\eta_{0}^{(2)}(s)=1-\epsilon(1-\hat{s} / s)^{3 / 2}, \\
\epsilon=0.18 \pm 0.12 \quad\left(\hat{s}^{1 / 2}=1.05 \mathrm{GeV}\right) .
\end{gathered}
$$

\section{The $P$ wave}

The region $s^{1 / 2} \leq 2 m_{K} \mathrm{GeV}$. We have now

$$
\begin{gathered}
B_{0}=1.052 \pm 0.011, \quad B_{1}=0.17 \pm 0.05, \\
M_{\rho}=773.6 \pm 0.9 \mathrm{MeV} .
\end{gathered}
$$

The $P$ wave between $2 m_{K}$ and $1420 \mathrm{MeV}$. The parameters are now

$$
\begin{gathered}
\lambda_{0}=2.684 \pm 0.009, \quad \lambda_{1}=1.50 \pm 0.18 \\
\lambda_{2}=-1.97 \pm 0.49, \quad \epsilon_{1}=0.09 \pm 0.06 \\
\epsilon_{2}=0.12 \pm 0.11
\end{gathered}
$$

\section{Parametrization of the DO wave}

The parameters of this wave do not differ appreciably from those in Appendix A. 


\section{Parametrization of the D2 wave}

We have now

$$
\begin{array}{ll}
B_{0}=(3.1 \pm 0.5) \times 10^{3}, & B_{1}=(7.9 \pm 1.0) \times 10^{3}, \\
B_{2}=(24.7 \pm 4.2) \times 10^{3}, & \Delta=205 \pm 25 \mathrm{MeV},
\end{array}
$$

and

$$
\epsilon=0.15 \pm 0.2 \text {. }
$$

\section{The $F$ wave}

The parameters of this wave do not differ appreciably from those in Appendix A.

\section{The $\mathbf{G}$ waves}

We have not varied the parameters of the $G$ waves, which therefore are as in Appendix A above.

\section{Regge parameters}

We here give the Regge parameters, obtained with the constrained fits. They are to be used with the formulas of Sec. III.

Isospin 0.

$$
\begin{gathered}
\beta_{P}=2.54 \pm 0.04, \quad c_{P}=0.53 \pm 1.0 \mathrm{GeV}^{-2}, \quad \alpha_{P}^{\prime}=0.20 \pm 0.10 \mathrm{GeV}^{-2} \\
\beta_{P^{\prime}}=0.83 \pm 0.05, \quad c_{P^{\prime}}=-0.38 \pm 0.4 \mathrm{GeV}^{-2}, \quad \alpha_{P^{\prime}}(0)=0.54 \pm 0.02, \quad \alpha_{P^{\prime}}^{\prime}=0.90 \mathrm{GeV}^{-2}
\end{gathered}
$$

Isospin 1.

$$
\begin{array}{lr}
\beta_{\rho}=1.30 \pm 0.14, & \alpha_{\rho}(0)=0.46 \pm 0.02, \\
\alpha_{\rho}^{\prime}=0.90 \mathrm{GeV}^{-2}, & \alpha_{\rho}^{\prime \prime}=-0.3 \mathrm{GeV}^{-4}, \\
d_{\rho}=2.4 \pm 0.5, & e_{\rho}=0.0 \pm 2.5 \mathrm{GeV}^{-4}
\end{array}
$$

Isospin 2.

$$
\beta_{2}=0.22 \pm 0.2
$$

\section{APPENDIX C: THE CONFORMAL MAPPING METHOD}

In this appendix we explain a few of the features of the method of conformal mapping expansion. Although it is a standard method for calculating analytic functions with cuts (for example, it is one of the methods used by computers to evaluate logarithms), we hope that devoting a few lines to the matter would not be a waste. To make the discussion more adapted to our case, we will exemplify our discussion with the S0 wave.

The key point in the method is the remark that the analyticity and unitarity properties of a $\pi \pi$ partial wave amplitude, ${ }^{12} f(s)$, imply analyticity of the effective range function, $\psi(s)$, given by (for the S0 wave)

$$
\cot \delta(s)=\frac{s^{1 / 2}}{2 k} \frac{M_{\pi}^{2}}{s-\frac{1}{2} z_{0}^{2}}\left\{\frac{z_{0}^{2}}{M_{\pi} \sqrt{s}}+\psi(s)\right\},
$$

in the full complex $s$ plane cut from $-\infty$ to 0 , and from $s_{0}=4 m_{\underline{K}}^{2}$ to $+\infty$; we are neglecting here inelasticity below the $\bar{K} K$ threshold. The function $\psi(s)$ is so constructed

\footnotetext{
${ }^{12}$ To lighten the notation we will, in the present appendix, suppress indices. The rest of the notation is as in the main text.
}

that it does not have the elastic cut. To find an expansion that respects this analyticity of $\psi$, we map the cut plane into a circle (Fig. 15), which is accomplished in our case by the change of variable (conformal mapping)

$$
s \rightarrow w(s)=\frac{\sqrt{s}-\sqrt{s_{0}-s}}{\sqrt{s}+\sqrt{s_{0}-s}} .
$$

Under the mapping, the left-hand cut is mapped into the left half unit circle, and the inelastic cut into the right half of the circle; see Fig. 16. The analyticity region (cut plane) is mapped into the interior of the unit circle. The function $\psi(w)$ is then analytic, in the variable $w$, in the unit disk: hence, the analyticity properties of $\psi$ are strictly equivalent to the convergence of the Taylor expansion, ${ }^{13}$

$$
\psi(w)=B_{0}+B_{1} w+B_{2} w^{2}+\cdots,
$$

in the unit disk, $|w|<1$.

Reverting to the variable $s$, the expansion (C3) becomes, for the cotangent of the phase shift, the expansion

$$
\begin{aligned}
& \cot \delta(s)=\frac{s^{1 / 2}}{2 k} \frac{M_{\pi}^{2}}{s-\frac{1}{2} z_{0}^{2}}\left\{\frac{z_{0}^{2}}{M_{\pi} \sqrt{s}}+B_{0}+B_{1} \frac{\sqrt{s}-\sqrt{s_{0}-s}}{\sqrt{s}+\sqrt{s_{0}-s}}\right. \\
& \left.+B_{2}\left(\frac{\sqrt{s}-\sqrt{s_{0}}-\bar{s}}{\sqrt{s}+\sqrt{s_{0}-s}}\right)^{2}+\cdots\right\} \text {. }
\end{aligned}
$$

In our work we only use the expansion in a small region, $|w| \lesssim 0.56$, which is away from the cuts (this will be discussed in more detail later); but it is not difficult to prove that $(\mathrm{C} 4)$ also represents the function $\cot \delta(s)$ on the cuts. We show this next.

\footnotetext{
${ }^{13}$ Other methods use mapping into an ellipse, and expansions in orthogonal polynomials; see, e.g., Ref. [21].
} 


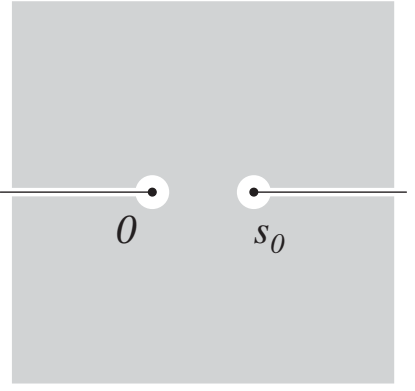

FIG. 15. The mapping $s \rightarrow w$.

We write a dispersion relation for the function $\psi^{\text {ex. }}(s)$, taken to be the exact function:

$$
\psi^{\text {ex. }}(s)=\frac{1}{\pi} \int_{-\infty}^{0} \mathrm{~d} s^{\prime} \frac{\operatorname{Im} \psi^{\text {ex. }}\left(s^{\prime}\right)}{s^{\prime}-s}+\int_{s_{0}}^{\infty} \mathrm{d} s^{\prime} \frac{\operatorname{Im} \psi^{\text {ex. }}\left(s^{\prime}\right)}{s^{\prime}-s} ;
$$

we neglect eventual subtractions that play no role here. It is convenient to rewrite this with a change of integration variable, $y=s_{0} /\left(2 s-s_{0}\right)$, so that we have

$$
\psi^{\text {ex. }}(s)=\frac{2 s_{0}}{\pi} \int_{-1}^{+1} \mathrm{~d} y \frac{1}{\left[2 s^{\prime}(y)-s_{0}\right]^{2}} \frac{\operatorname{Im} \psi^{\text {ex. }}\left(s^{\prime}(y)\right)}{s^{\prime}(y)-s} .
$$

In this new variable, the left-hand cut is transformed in the interval $[-1,0]$, and the inelastic cut in $[0,+1]$.

Next, it is clear from the presence of the functions $\sqrt{s}$, $\sqrt{s}=s_{0}$ that $\cot \delta(s)$, or $\psi(s)$, have the correct left- and right-hand cuts. We write a dispersion relation for the function expanded to order $N, \psi^{N}(s)=\sum_{0}^{N} B_{n} w^{n}$; since it has cuts in the same places as the exact function, it will read

$$
\psi^{N}(s)=\frac{2 s_{0}}{\pi} \int_{-1}^{+1} \mathrm{~d} y \frac{1}{\left[2 s^{\prime}(y)-s_{0}\right]^{2}} \frac{\operatorname{Im} \psi^{N}\left(s^{\prime}(y)\right)}{s^{\prime}(y)-s} .
$$

Now, we identify $\psi^{N}(s)$ and $\psi^{\text {ex. }}(s)$ at a set of $\nu$ experimental points, $s_{j}$ (we neglect experimental errors), comprised in the interval $\left[4 M_{\pi}^{2}, s_{0}\right]: \psi^{N}\left(s_{j}\right)=\psi^{\text {ex. }}\left(s_{j}\right)$, $j=1, \ldots \nu$. This means that the functions on the cut have the same averages with the set of functions

$$
\begin{gathered}
\varphi_{j}(y)=\frac{1}{\left[2 s^{\prime}(y)-s_{0}\right]^{2} s^{\prime}(y)-s_{j}} ; \\
\int_{-1}^{+1} \mathrm{~d} y \varphi_{j}(y)\left\{\operatorname{Im} \psi^{N}\left(s^{\prime}(y)\right)-\operatorname{Im} \psi^{\text {ex. }}\left(s^{\prime}(y)\right)\right\}=0, \\
j=1, \ldots, \nu .
\end{gathered}
$$

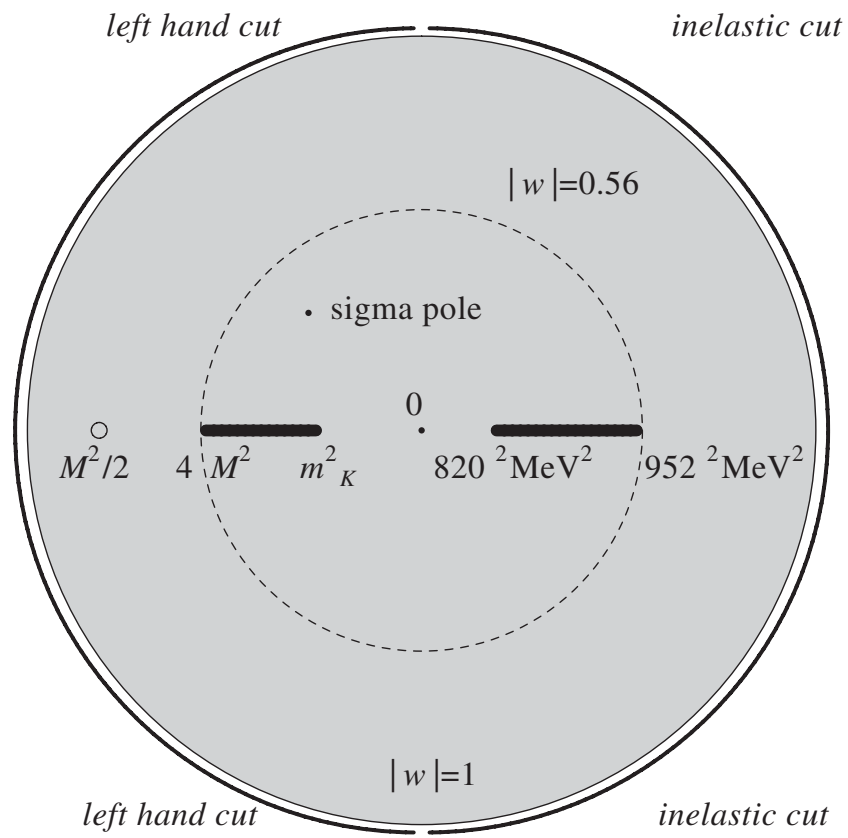

FIG. 16. The $w$ disk, $|w|<1$. The dashed line is the line $|w|=$ 0.56 . The thick lines are the regions where one has reliable experimental data (for the S0 wave). Images of the left-hand cut and of the inelastic cut are depicted. The location of the sigma pole is also shown.

Hence, the functions built with the $\psi^{N}$ converge to the $\psi^{\text {ex. }}$, corresponding to the exact partial wave amplitude, both on the left-hand cut and on the right-hand (inelastic) cut, in the mean. In the limit in which one had an infinite number of experimental points, the function $\psi^{\text {ex. }}$ would be represented exactly, because the set of functions in (C7) form a complete set in the interval $[-1,+1]$ (for a proof of this in a physical context, cf. Ref. [22]). In our case we have only a finite number of points ( 31 for the S0 case) and, moreover, they have experimental errors, so the representation on the cuts is valid only in the mean, and up to experimental errors.

In our applications, however, this convergence on the cuts is irrelevant, as we are only fitting experimental data, which are located in a region away both from the left-hand cut and the inelastic cut: cf. Fig. 16, where we represent the experimental data we are fitting in the case of the S0 wave (to the left we have the data obtained from $\mathrm{Ke} 4$ decays, and $K_{2 \pi}$ decays, and, to the right, those higher energy $\pi \pi$ scattering data points included in our fits). All of them fall inside the circle $|w|<0.56$. 
[1] J. R. Peláez and F. J. Ynduráin, Phys. Rev. D 71, 074016 (2005).

[2] R. Kamiński, J. R. Peláez, and F. J. Ynduráin, Phys. Rev. D 74, 014001 (2006); 74, 079903(E) (2006).

[3] R. García Martín, J.R. Peláez, and F. J. Ynduráin, Phys. Rev. D 76, 074034 (2007).

[4] K14 decays: L. Rosselet et al., Phys. Rev. D 15, 574 (1977); S. Pislak et al., Phys. Rev. Lett. 87, 221801 (2001); NA48/2 (CERN/SPS experiment); B. BlochDevaux, QCD06, Montpellier (France), 2006; L. Masetti, ICHEP06, Moscow (Russia), 2006. $K \rightarrow 2 \pi d e-$ cays: A. Aloisio et al., Phys. Lett. B 538, 21 (2002); C. Gatti and V. Cirigliano (private communication).

[5] S. M. Roy, Phys. Lett. 36B, 353 (1971).

[6] B. Hyams et al., Nucl. Phys. B64, 134 (1973); P. Estabrooks and A.D. Martin, Nucl. Phys. B79, 301 (1974); G. Grayer et al., Nucl. Phys. B75, 189 (1974); S. D. Protopopescu et al., Phys. Rev. D 7, 1279 (1973); R. Kamiński, L. Lesniak, and K. Rybicki, Z. Phys. C 74, 79 (1997); Eur. Phys. J. direct C 4, 1 (2002); B. Hyams et al., Nucl. Phys. B100, 205 (1975); M. J. Losty et al., Nucl. Phys. B69, 185 (1974); W. Hoogland et al., Nucl. Phys. B 126, 109 (1977); N. B. Durusoy et al., Phys. Lett. B 45, 517 (1973).

[7] $\pi \pi \rightarrow \bar{K} K$ scattering: W. Wetzel et al., Nucl. Phys. B115, 208 (1976); D. Cohen et al., Phys. Rev. D 22, 2595 (1980); E. Etkin et al., Phys. Rev. D 25, 1786 (1982).

[8] J.F. de Trocóniz and F. J. Ynduráin, Phys. Rev. D 65, 093001 (2002); 71, 073008 (2005).

[9] J. R. Peláez and F. J. Ynduráin, Phys. Rev. D 69, 114001 (2004). See also J. R. Cudell et al., Phys. Lett. B 587, 78 (2004); J. R. Peláez, arXiv: hep-ph/0510005. Note, however, that the first reference fits data for $\pi N$ and $N N$, but not for $\pi \pi$, and only for energies above $\sim 4 \mathrm{GeV}$; while the last article contains only preliminary results and, indeed, the parameters for exchange of isospin zero are not well determined.

[10] W. Rarita et al., Phys. Rev. 165, 1615 (1968).

[11] C. D. Froggatt and J. L. Petersen, Nucl. Phys. B129, 89 (1977).

[12] R. Kamiński, J. R. Peláez, and F. J. Ynduráin, Fourth International Conference on Quarks and Nuclear Physics, Madrid, 2006; Eur. Phys. J. A 31, 479 (2007).

[13] B. Ananthanarayan et al., Phys. Rep. 353, 207 (2001).

[14] S. L. Adler and F. J. Ynduráin, Phys. Rev. D 75, 116002 (2007).

[15] B. Adeva, A. Romero Vidal, and O. Vázquez Doce, Eur. Phys. J. A 31, 522 (2007).

[16] N. Cabibbo and G. Isidori, J. High Energy Phys. 03 (2005) 021; NA48 Experiment: see, e.g., S. Balev, arXiv:0705.4183v2.

[17] G. Colangelo, J. Gasser, and H. Leutwyler, Nucl. Phys. B603, 125 (2001).

[18] A. Dobado and J. R. Peláez, Phys. Rev. D 56, 3057 (1997); J. A. Oller, E. Oset, and J. R. Peláez, Phys. Rev. D 59, 074001 (1999); 60, 099906 (1999); 75, 099903 (2007); A. Gómez-Nicola and J. R. Peláez, Phys. Rev. D 65, 054009 (2002).

[19] J. R. Peláez and F. J. Ynduráin, Phys. Rev. D 68, 074005 (2003).

[20] J. Gasser, arXiv:0710.3048.

[21] S. Ciulli, Strong Interactions, Lecture Notes in Physics (Springer-Verlag, New York, 1973); J. Pišut, Low Energy Hadron Interactions (Springer, Berlin 1970).

[22] F. J. Ynduráin, Ann. Phys. (N.Y.) 75, 171 (1973). 